\title{
Search for Low-Mass Quark-Antiquark Resonances Produced in Association with a Photon at $\sqrt{s}=13 \mathrm{TeV}$
}

\author{
A. M. Sirunyan et al. \\ (CMS Collaboration)
}

(Received 24 May 2019; revised manuscript received 12 October 2019; published 3 December 2019)

\begin{abstract}
A search for narrow low-mass resonances decaying to quark-antiquark pairs is presented. The search is based on proton-proton collision events collected at $13 \mathrm{TeV}$ by the CMS detector at the CERN LHC. The data sample corresponds to an integrated luminosity of $35.9 \mathrm{fb}^{-1}$, recorded in 2016 . The search considers the case where the resonance has high transverse momentum due to initial-state radiation of a hard photon. To study this process, the decay products of the resonance are reconstructed as a single large-radius jet with two-pronged substructure. The signal would be identified as a localized excess in the jet invariant mass spectrum. No evidence for such a resonance is observed in the mass range 10 to $125 \mathrm{GeV}$. Upper limits at the $95 \%$ confidence level are set on the coupling strength of resonances decaying to quark pairs. The results obtained with this photon trigger strategy provide the first direct constraints on quark-antiquark resonance masses below $50 \mathrm{GeV}$ obtained at a hadron collider.
\end{abstract}

DOI: 10.1103/PhysRevLett.123.231803

New resonances coupling to pairs of quarks (generally referred to as $Z^{\prime}$ ) are ubiquitous signatures in theories beyond the standard model (SM), appearing in dark matter models [1,2] and models with extra dimensions [3], among others [4-9]. The first dijet searches at a hadron collider were performed by UA1 [10] and UA2 [11], and have been extended to higher resonance masses by $\mathrm{CDF}$ [12] and D0 [13] at the Tevatron, and by ATLAS [14] and CMS [15] at the LHC. However, as collision energy and beam intensity have increased, there has been a loss of sensitivity to lower mass resonances, which stems from the increasing cross section of background multijet events, tighter online requirements needed to handle growing event rates, and the large numbers of simultaneous collisions per bunch crossing (pileup). These issues can be partially mitigated by focusing on events in which the resonance is produced in association with high momentum initial-state radiation (ISR). In such a scenario, the two quarks hadronize into a single massive jet. In particular, by considering events with a high transverse momentum $\left(p_{T}\right)$ ISR photon or jet, the ATLAS Collaboration searched for $Z^{\prime}$ decaying to quark-antiquark pairs [16] and reported a result for resonance masses as low as $100 \mathrm{GeV}$. The CMS Collaboration used this method with ISR jets to search for $Z^{\prime}$ with masses

\footnotetext{
*Full author list given at the end of the article.

Published by the American Physical Society under the terms of the Creative Commons Attribution 4.0 International license. Further distribution of this work must maintain attribution to the author(s) and the published article's title, journal citation, and DOI. Funded by SCOAP.
}

as low as $50 \mathrm{GeV}$ [17], the lowest mass then probed by collider experiments.

This analysis, which considers events produced with ISR photons from $p p$ collisions at $\sqrt{s}=13 \mathrm{TeV}$, using data collected by the CMS detector in 2016, and corresponding to an integrated luminosity of $35.9 \mathrm{fb}^{-1}$, extends dijet searches to low $Z^{\prime}$ masses where only indirect measurements [18] provide constraints on the hadronic production of such new physics. This extension to low $Z^{\prime}$ masses is possible in this analysis because of the reliance on a photon trigger, for which it is feasible to select dijet events using a lower $p_{T}$ threshold than for jet triggers. However, the mass of the $Z^{\prime}$ is sufficiently low compared to its momentum that the separate hadronizations of the resulting quark and antiquark merge into a single large-radius jet. This search is performed by looking for a localized excess in the jet mass spectrum in events with a photon and a jet with the two-pronged jet substructure expected for the signal.

The main background, arising from photons produced in association with jets by SM processes, is derived using a data-driven method. Additional resonant SM background processes, composed of $t \bar{t}$ events and the SM production of $W+\gamma$ and $Z+\gamma$, are estimated from simulation, with corrections obtained from control regions in data. The results are interpreted within the framework of a $Z^{\prime}$ with mass between 10 and $125 \mathrm{GeV}$, decaying into quarks, and are used to set limits on the quark coupling $g_{q}^{\prime}$ as a function of the $Z^{\prime}$ mass.

The CMS detector consists of a silicon tracker, a lead tungstate crystal electromagnetic calorimeter (ECAL), a brass and scintillator hadron calorimeter (HCAL), and gasionization muon detectors. A superconducting solenoid provides a uniform magnetic field within the detector. 
Events are sorted by a two-tiered triggering system [19] to ensure that only events of potential physics interest are recorded. A more detailed description of the CMS detector, including its angular coordinates $\eta$ and $\phi$, can be found in Ref. [20].

Events are reconstructed using the CMS particle-flow (PF) [21] algorithm, which combines information from every element of the CMS detector to reconstruct and identify individual particles (called PF candidates). Each particle is classified as either a muon, electron, photon, charged hadron, or neutral hadron. The energy of photons is obtained directly from ECAL measurements. Similar measurements, along with information from the tracker, are used to determine the energy of electrons. Misidentification of particles is possible, so additional isolation and purity requirements on potential photons are imposed [22]. The momentum of muons is measured from the curvature of their tracks. Neutral and charged hadron energies are measured from their deposits in the ECAL and HCAL, with information from the tracker used to further constrain the energy of the charged hadrons. The missing transverse momentum $\left(\vec{p}_{\mathrm{T}}^{\text {miss }}\right)$ is defined as the negative vector $p_{T}$ sum of all reconstructed particles in an event. The PF candidates are clustered into jets using FastJet [23] with the anti- $k_{T}$ algorithm [24] and a distance parameter of 0.4 and 0.8 for AK4 and AK8 jets, respectively. Particles produced in additional collisions within the same bunch crossing are suppressed by applying a weight to each PF candidate, calculated by the pileup-per-particle identification [25] algorithm. Jets are corrected as a function of their $p_{T}$ and pseudorapidity $(\eta)$ to match the observed detector response [26]. Jets arising from the hadronization of $b$ quarks are identified using the CSVv2 algorithm [27].

The signal benchmark model [28] used in this analysis and in Refs. [16,17] features a vector resonance $Z^{\prime}$, with the coupling constant to quarks set to $g_{q}^{\prime}=1 / 6$, at which the $Z^{\prime}$ width is well below the resolution of the detector. It was simulated to leading order with the MADGRAPH5_aMC@NLO [29] generator, with MLM matching [30] between jets from matrix element calculations and the parton showers. Up to 3 additional jets are allowed in the matrix element calculation. The model assumes no interaction between the SM $Z$ and the $Z^{\prime}$. The same generator is used to model at leading order the quantum chromodynamic production of multijet events, which can include radiated photons, and the $\gamma+$ jets background, where the photon is part of the hard interaction, as well as to next-to-leading order, the backgrounds $W+\gamma$ and $Z+\gamma$. The multijet and $\gamma+$ jet components are treated together as a single nonresonant background, with the angle between the leading photon and the nearest jet used to define a phase space for each sample. Events from the multijet sample are removed if they are in the $\gamma+$ jets phase space. The POWHEG 2.0 [3133 ] generator is used to model $t \bar{t}$ events at next-to-leading order. All signal and background generators are interfaced with PYTHIA 8.212 [34], with the CUETP8M1 underlying event tune [35], to simulate parton showering and hadronization effects. The generated events are processed through a GEANT4 [36] simulation of the CMS detector. This simulation includes effects from both in-time and out-of-time pileup. The parton distribution function set NNPDF3.0 [37] is used to produce all simulated samples. Where necessary, differences between the reconstruction of simulated and real quantities are corrected by applying scale factors to the simulation, derived from control regions in data [26].

The trigger strategy used by this search is to require one photon with $p_{T}>175 \mathrm{GeV}$ and $|\eta|<3.0$. To ensure a full triggering efficiency for events that satisfy the subsequent selection, offline photons are required to have $p_{T}>$ $200 \mathrm{GeV}$ and $|\eta|<2.4$. Events with additional identified photons of $p_{T}>14$ or leptons of $p_{T}>10 \mathrm{GeV}$ are discarded to avoid overlap with other searches and to reduce backgrounds from electroweak sources. Even leptons in a pair that are sufficiently colinear to be reconstructed as a single jet are generally also tagged as separate leptons and thus excluded. The $Z^{\prime} \rightarrow q \bar{q}$ decay is assumed to correspond to the highest momentum AK8 jet in the event. Only events with leading jet $p_{T}>200 \mathrm{GeV}$ are considered. To reduce the contribution from $t \bar{t}$, events with an AK4 jet with $p_{T}>$ $30 \mathrm{GeV}$ and satisfying the loose working point [27] of the CSVv2 algorithm (excluding AK4 jets within $\Delta R<0.6$ of the leading AK8 jet), or with $p_{T}^{\text {miss }}>75 \mathrm{GeV}$, are discarded. A separation $\sqrt{(\Delta \eta)^{2}+(\Delta \phi)^{2}}>2.2$ is required between the leading AK8 jet and the photon in the event.

The soft drop mass algorithm (with $\beta=0, z_{\text {cut }}=0.1$ ) $[38,39]$ is used to remove soft and wide-angle radiation from the jet, and the resulting distribution of "groomed jet mass" $\left(m_{\mathrm{SD}}\right)$ is inspected for localized excesses. The modeling of $m_{\mathrm{SD}}$ has been tested for masses only down to $10 \mathrm{GeV}$ [40]; thus $10 \mathrm{GeV}$ is the lowest signal mass considered by this analysis. The highest signal mass considered is $125 \mathrm{GeV}$, above which there is a low probability of reconstructing the $Z^{\prime}$ as a single jet. The selected events are divided into signal and control regions based on the $\eta$ of the photon, with the boundary between the regions chosen to maximize the sensitivity of the analysis. Events with photon $|\eta|<2.1$ are considered to be in the signal region. The events with $|\eta|>2.1$, in which the photon is more likely to have been radiated in a multijet process rather than in a hard scattering, define the $\eta$ control region to perform substructure measurements of jets with kinematic variables similar to those of jets in the signal region. These variables are computed only on jet constituents that have survived the soft drop algorithm.

The variable $N_{2}^{1}[41,42]$ is used to further separate signal jets with two-pronged substructure from the background. This variable is defined using a combination of functions that correlate angles among the constituents of the jet to categorize the substructure. A jet originating from a twopronged decay is more likely to have a low value of $N_{2}^{1}$. In 


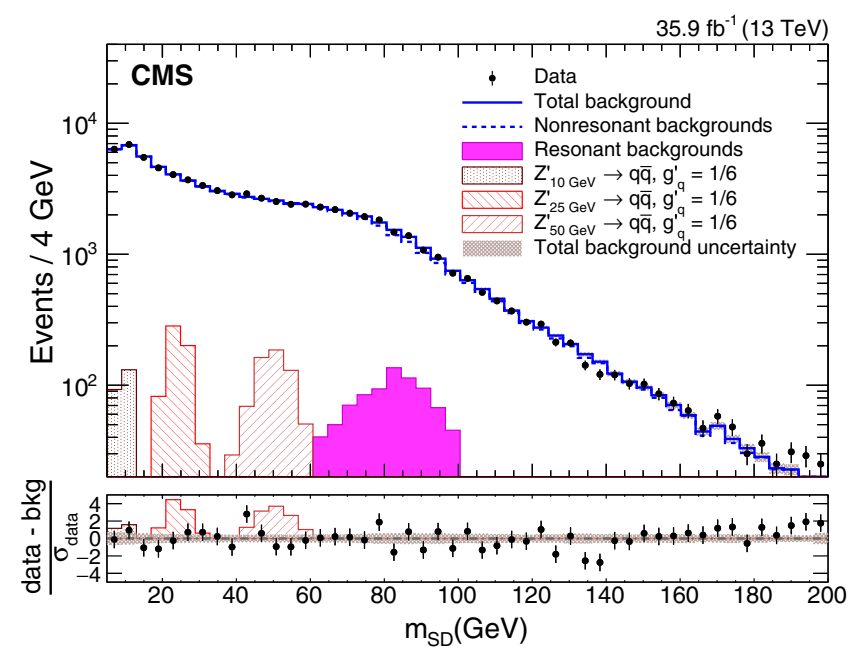

FIG. 1. The soft drop [38,39] jet mass distribution of the signal region after the main background estimation fit is performed. The nonresonant background is indicated by a dashed line, while the total background composed of the sum of this nonresonant background and the resonant backgrounds is shown by the solid line. Representative signals are plotted for comparison. The bottom panel shows the difference between the data and the final background estimate, divided by the statistical uncertainty of the data in each bin. The shaded region represents the total uncertainty in the background estimate in each bin.

addition, we define the dimensionless quantity $\rho=$ $\ln \left(m^{2} / p_{T}^{2}\right)$ [43], which, unlike the mass itself, is approximately uncorrelated with the jet $p_{T}$.

While the $N_{2}^{1}$ variable offers a considerable discrimination power, the background efficiency for retaining jets based on a fixed cut on $N_{2}^{1}$ has dependencies on the jet $\rho$ and $p_{T}$. These lead to distortion of the $m_{\mathrm{SD}}$ distribution, making a search for a peak difficult. To preserve the shape of the mass distribution, a varying cut on $N_{2}^{1}$ is used to remove $90 \%$ of the background. To achieve this, a decorrelated variable $N_{\mathrm{DDT}}^{2}$ is built, which is similar to the one proposed in Ref. [43]. This variable is defined as

$$
N_{\mathrm{DDT}}^{2}\left(\rho^{\mathrm{jet}}, p_{T}^{\mathrm{jet}}\right)=N_{2}^{1}-X_{10 \%}\left(\rho^{\mathrm{jet}}, p_{T}^{\mathrm{jet}}\right),
$$

where $X_{10 \%}$ is the value of $N_{2}^{1}$ where a cut would retain $10 \%$ of the background. The values for $X_{10 \%}$ in bins of the jet $\rho$ and $p_{T}$ are taken from the $\eta$ control region. A smoothing procedure is applied to the $X_{10 \%}$ distribution to reduce unphysical features where the statistical uncertainty is large. The selection $N_{\mathrm{DDT}}^{2}<0$ is applied to events in the signal region. By construction, this selection will have a background efficiency of exactly $10 \%$ for the sample in which $N_{\text {DDT }}^{2}$ was constructed. Signal hypotheses across the entire parameter range of the analysis were injected into simulated background distributions to evaluate potential contamination of the $\eta$ control region and its effect on the mass distribution of events passing the $N_{\text {DDT }}^{2}<0$ requirement. The effects were found to be negligible compared to the statistical uncertainty in the mass distribution. Differences in the $p_{T}$ and $\rho$ dependence of $X_{10 \%}$ in the signal and control regions are expected, and are explicitly parametrized as part of the background estimation procedure.

The dominant background is due to nonresonant events in which a light quark or gluon jet passes the $N_{\text {DDT }}^{2}$ requirement. The second component consists of events with two-pronged jets arising from a mixture of $Z+\gamma$, $W+\gamma$, and $t \bar{t}$ events. Events from $t \bar{t}$ production enter the signal selection largely through electrons being incorrectly reconstructed as photons. Other sources of background were found to be negligible. The nonresonant background is estimated from a data-driven method described below, with the simulated samples used for validation only. The other backgrounds are taken from simulation and their shapes and normalizations allowed to vary in a final fit of the passing and failing regions, with a correction derived from a $t \bar{t}$ control region, also described below.

The nonresonant background in the signal region is estimated by considering the events that have passed all selection requirements except $N_{\text {DDT }}^{2}<0$. In the $\eta$ control region, the pass-to-fail ratio of background events for the $N_{\text {DDT }}^{2}<0$ requirement is one to nine, independent of jet $p_{T}$ and $\rho$. In the signal region, this ratio is taken to be a smooth function $\mathcal{F}\left(p_{T}, \rho\right)$ which models the differences between the $N_{\text {DDT }}^{2}$ variable in the $\eta$ control and signal regions. The relationship between passing $\left(N_{\mathrm{P}}\right)$ and failing $\left(N_{\mathrm{F}}\right)$ events is then $N_{\mathrm{P}}=\mathcal{F}\left(p_{T}, \rho\right) N_{\mathrm{F}}$, for each bin of $p_{T}$ and $\rho$. The deviation of $\mathcal{F}$ from a flat ratio corresponds to the difference between the signal and control regions. The unknown function $\mathcal{F}$ is expanded into a polynomial series:

$$
\mathcal{F}\left(p_{T}, \rho\right)=\sum_{i} \sum_{j} a_{i j} p_{T}^{j} \rho^{i},
$$

where the unknown coefficients $a_{i j}$ are determined by a simultaneous likelihood fit of the passing and failing events, in which the signal and resonant backgrounds are allowed to float. The number of coefficients in the fit is determined by performing the Fisher $F$ tests [44] on progressively higher order polynomial combinations of $p_{T}$ and $\rho$. The optimal polynomial form is found to be third order in both $p_{T}$ and $\rho$.

While this fit to data ensures that differences in the nonresonant background modeling of the $N_{\mathrm{DDT}}^{2}$ variable are accounted for, consistent behavior in data and simulation for resonances is not assured. A dedicated $t \bar{t}$ control region is defined, built from events containing a high- $p_{T}$ muon, with the selection optimized to be dominated by $t \bar{t}$ production. The efficiency of the $N_{\mathrm{DDT}}^{2}<0$ requirement is measured by fitting the $W$-mass peak (where the hadronization products from both quarks merge into one jet) in the passing and failing jet mass distributions of this control region, for both data and simulated samples. This efficiency, an explicit parameter of the fit, is used to correct relative yields for 
resonant $t \bar{t}$ events and the $W+\gamma$ and $Z+\gamma$ backgrounds obtained from simulation in the passing and failing regions. The data-to-simulation efficiency scale factor is found to be $0.909 \pm 0.046$ (stat + syst), and is applied to all the resonant backgrounds, as well as to the signal.

To model the $m_{\mathrm{SD}}$ distribution in the signal region, a binned 2D maximum likelihood fit is performed on the events passing and failing the $N_{\mathrm{DDT}}^{2}<0$ requirement, in all $\left(p_{T}, \rho\right)$ bins of the signal region [17]. In the fit, all SM processes and the signal are allowed to float simultaneously. Signal shapes are taken from simulation. The fit is performed for the background-only (null) hypothesis and for signal hypotheses for each simulated signal mass (10, $25,50,75,100$, and $125 \mathrm{GeV}$ ), as well as for interpolated mass shapes derived by vertical template morphing [45] these simulated event distributions to cover a signal hypothesis in steps of $5 \mathrm{GeV}$ from 10 to $125 \mathrm{GeV}$. To ensure proper modeling of the high mass tail, the fit is performed on events with masses up to $201 \mathrm{GeV}$. The $m_{\mathrm{SD}}$ distribution of the signal region, summed over all $p_{T}$ and $\rho$ bins, is shown in Fig. 1. The contributions from resonant backgrounds are evaluated as part of the likelihood, with their shapes and normalizations allowed to vary within the systematic uncertainties in the initial estimates (see Table I). The average value of the nonresonant background efficiency in the signal region determined by the fit is $9 \%$.

The uncertainty in the nonresonant background originates from the systematic uncertainty in the fit and the statistical uncertainty from the number of events in the region failing the $N_{\mathrm{DDT}}^{2}<0$ requirement. The signal, $t \bar{t}$, $W+\gamma$, and $Z+\gamma$ backgrounds are affected by correlated shape and normalization uncertainties. We constrain the efficiency of the selection based on $N_{\mathrm{DDT}}^{2}$ in the $t \bar{t}$ control region, with the scale factor uncertainty applied to the yields of signal and the resonant backgrounds in the final fit to the signal region. The jet mass scale and resolution uncertainties are considered as uncertainties in the shape of the signal and the resonant background components in the fit. Finally, uncertainties associated with the jet energy corrections [26], trigger efficiency, lepton veto efficiency, resonant background normalizations and the integrated luminosity determination [46] are applied to the expected yields of the signal and the resonance backgrounds. These are summarized in Table I. To validate the robustness of the fit, a goodness-of-fit test and bias tests are performed using simulated data with a variety of simulated signals injected. No significant bias is observed for any $Z^{\prime}$ mass.

The results of the fit are used to set $95 \%$ confidence level (C.L.) upper limits on $g_{q}^{\prime}$. Upper limits are computed under a modified frequentist approach, using the $\mathrm{CL}_{\mathrm{s}}$ criterion $[47,48]$. A profile likelihood ratio is used as the test statistic and its distribution under the null and alternate hypotheses are determined with asymptotic approximations [49]. Limits are shown in Fig. 2 as a function of the resonance mass. Coupling values above the solid curves are excluded
TABLE I. The systematic uncertainties included in the computation of the limit on the coupling strength of $Z^{\prime}$ to quarks. Parameters denoted by the $\star$ symbol affect both the shape and normalization of the affected processes; otherwise only the normalization is modified. The parameters affecting normalizations have log-normal priors, and those affecting the shape have Gaussian priors, unless marked with the $\uparrow$ symbol, which denotes that this parameter was floating and constrained by the final simultaneous fit of the passing and failing distributions.

\begin{tabular}{|c|c|c|}
\hline Systematic effect & Affected processes & Uncertainty (\%) \\
\hline Polynomial fit ${ }^{\dagger \star}$ & Nonresonant & $1-5$ \\
\hline Electron veto & $t \bar{t}, W, Z, Z^{\prime}$ & 0.5 \\
\hline Muon veto & $\overline{t \bar{t}}, W, Z, Z^{\prime}$ & 0.5 \\
\hline Jet mass smear ${ }^{\dagger \star}$ & $t \bar{t}, W, Z, Z^{\prime}$ & 0.7 \\
\hline Jet energy corrections & $t \bar{t}, W, Z, Z^{\prime}$ & 2 \\
\hline Luminosity & $\bar{t} \bar{t}, W, Z, Z^{\prime}$ & 2.5 \\
\hline Trigger $^{\star}$ & $\bar{t} \bar{t}, W, Z, Z^{\prime}$ & 3 \\
\hline$N_{\text {DDT }}^{2}$ efficiency & $t \bar{t}, W, Z, Z^{\prime}$ & 5 \\
\hline Photon ID & $t \bar{t}, W, Z, Z^{\prime}$ & 6 \\
\hline Jet mass scale ${ }^{\dagger \star}$ & $t \bar{t}, W, Z, Z^{\prime}$ & 6 \\
\hline$W+\gamma$ normalization $^{\dagger}$ & $W$ & 11 \\
\hline$Z+\gamma$ normalization $^{\dagger}$ & $Z$ & 45 \\
\hline$t \bar{t}$ normalization ${ }^{\dagger}$ & $t \bar{t}$ & 54 \\
\hline
\end{tabular}

at $95 \%$ C.L. Systematic uncertainties are treated as nuisance parameters, which are modeled with log-normal priors and profiled in the limit calculations. Values of $g_{q}^{\prime}$ greater than 0.3 are excluded at $95 \% \mathrm{CL}$ for the entire mass range. For most of the mass range below $50 \mathrm{GeV}$, made accessible by the trigger strategy, the exclusion from this analysis is more stringent than the indirect limits set by measurements of the $Z$ boson and $\Upsilon$ meson decay widths [18].

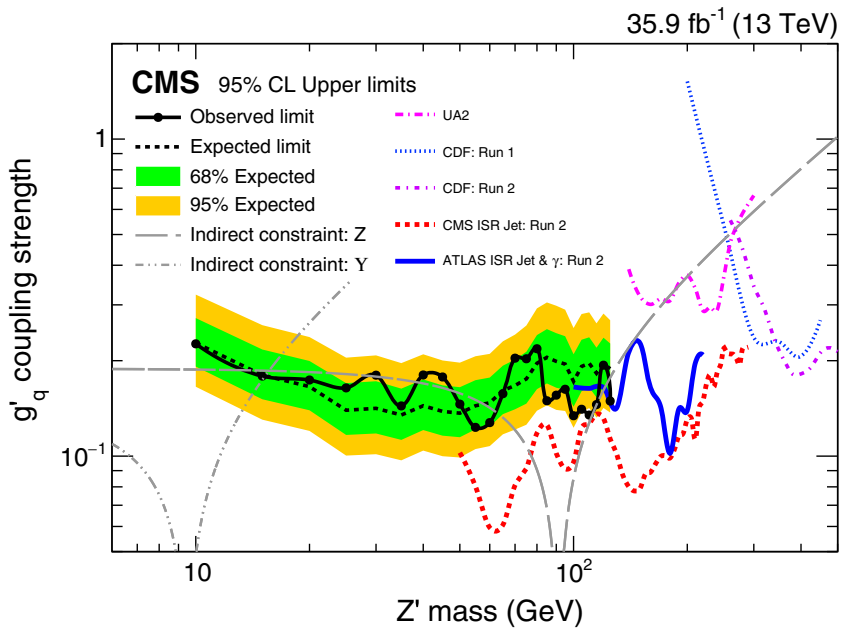

FIG. 2. Upper limits at 95\% C.L. on the coupling strength $g_{q}^{\prime}$ of $Z^{\prime} \rightarrow q \bar{q}$. The observed limit is shown as a solid black line, while the expected limit is dashed. The green (dark) and yellow (light) bands represent 1 and 2 standard deviation intervals. Limits from other searches and the indirect constraint from measurements of the $\Upsilon$ and $Z$ boson decay widths [18] are also shown. 
In summary, a search for a low mass $Z^{\prime}$ resonance decaying to $q \bar{q}$ pairs has been presented, using data from proton-proton collisions at the LHC with a center-of-mass energy of $13 \mathrm{TeV}$. Jet substructure and decorrelation techniques are implemented to search for narrow resonances over a smoothly falling background of the jet groomed mass. No significant excess is observed above the standard model expectation. Upper limits are placed on the quark coupling strength $g_{q}^{\prime}$ of $Z^{\prime}$ bosons with masses between 10 and $125 \mathrm{GeV}$. Below $50 \mathrm{GeV}$, the results obtained with this trigger strategy probe the lowest diquark resonance masses reached by a hadron collider.

We congratulate our colleagues in the CERN accelerator departments for the excellent performance of the LHC and thank the technical and administrative staffs at CERN and at other CMS institutes for their contributions to the success of the CMS effort. In addition, we gratefully acknowledge the computing centers and personnel of the Worldwide LHC Computing Grid for delivering so effectively the computing infrastructure essential to our analyses. Finally, we acknowledge the enduring support for the construction and operation of the LHC and the CMS detector provided by the following funding agencies: BMBWF and FWF (Austria); FNRS and FWO (Belgium); CNPq, CAPES, FAPERJ, FAPERGS, and FAPESP (Brazil); MES (Bulgaria); CERN; CAS, MoST, and NSFC (China); COLCIENCIAS (Colombia); MSES and CSF (Croatia); RPF (Cyprus); SENESCYT (Ecuador); MoER, ERC IUT, PUT, and ERDF (Estonia); Academy of Finland, MEC, and HIP (Finland); CEA and CNRS/IN2P3 (France); BMBF, DFG, and HGF (Germany); GSRT (Greece); NKFIA (Hungary); DAE and DST (India); IPM (Iran); SFI (Ireland); INFN (Italy); MSIP and NRF (Republic of Korea); MES (Latvia); LAS (Lithuania); MOE and UM (Malaysia); BUAP, CINVESTAV, CONACYT, LNS, SEP, and UASLP-FAI (Mexico); MOS (Montenegro); MBIE (New Zealand); PAEC (Pakistan); MSHE and NSC (Poland); FCT (Portugal); JINR (Dubna); MON, RosAtom, RAS, RFBR, and NRC KI (Russia); MESTD (Serbia); SEIDI, CPAN, PCTI, and FEDER (Spain); MOSTR (Sri Lanka); Swiss Funding Agencies (Switzerland); MST (Taipei); ThEPCenter, IPST, STAR, and NSTDA (Thailand); TUBITAK and TAEK (Turkey); NASU and SFFR (Ukraine); STFC (United Kingdom); DOE and NSF (USA).

[1] D. Abercrombie et al., Dark matter benchmark models for early LHC Run-2 searches: Report of the ATLAS/CMS dark matter forum, Phys. Dark Universe 26, 100371 (2019).

[2] G. Busoni et al., Recommendations on presenting LHC searches for missing transverse energy signals using simplified $s$-channel models of dark matter, arXiv:1603.04156.
[3] L. Randall and R. Sundrum, An Alternative to Compactification, Phys. Rev. Lett. 83, 4690 (1999).

[4] L. A. Anchordoqui, H. Goldberg, D. Lüst, S. Nawata, S. Stieberger, and T. R. Taylor, Dijet Signals for Low Mass Strings at the Large Hadron Collider, Phys. Rev. Lett. 101, 241803 (2008).

[5] S. Cullen, M. Perelstein, and M. E. Peskin, TeV strings and collider probes of large extra dimensions, Phys. Rev. D 62 , 055012 (2000).

[6] P. H. Frampton and S. L. Glashow, Chiral color: An alternative to the standard model, Phys. Lett. 190B, 157 (1987).

[7] R. S. Chivukula, A. Farzinnia, E. H. Simmons, and R. Foadi, Production of massive color-octet vector bosons at next-to-leading order, Phys. Rev. D 85, 054005 (2012).

[8] E. H. Simmons, Coloron phenomenology, Phys. Rev. D 55, 1678 (1997).

[9] U. Baur, M. Spira, and P. M. Zerwas, Excited quark and lepton production at hadron colliders, Phys. Rev. D 42, 815 (1990).

[10] C. Albajar et al. (UA1 Collaboration), Two jet mass distributions at the CERN proton-anti-proton collider, Phys. Lett. B 209, 127 (1988).

[11] J. Alitti et al. (UA2 Collaboration), A search for new intermediate vector mesons and excited quarks decaying to two jets at the CERN $\bar{p} p$ collider, Nucl. Phys. B400, 3 (1993).

[12] T. Aaltonen et al. (CDF Collaboration), Search for new particles decaying into dijets in proton-antiproton collisions at $\sqrt{s}=1.96 \mathrm{TeV}$, Phys. Rev. D 79, 112002 (2009).

[13] V. Abazov et al. (D0 Collaboration), Search for new particles in the two jet decay channel with the D0 detector, Phys. Rev. D 69, 111101 (2004).

[14] ATLAS Collaboration, Search for new phenomena in dijet events using $37 \mathrm{fb}^{-1}$ of $p p$ collision data collected at $\sqrt{s}=$ $13 \mathrm{TeV}$ with the ATLAS detector, Phys. Rev. D 96, 052004 (2017).

[15] CMS Collaboration, Search for narrow and broad dijet resonances in proton-proton collisions at $\sqrt{s}=13 \mathrm{TeV}$ and constraints on dark matter mediators and other new particles, J. High Energy Phys. 08 (2018) 130.

[16] ATLAS Collaboration, Search for low-mass resonances decaying into two jets and produced in association with a photon using $p p$ collisions at $\sqrt{s}=13 \mathrm{TeV}$ with the ATLAS detector, Phys. Lett. B 788, 316 (2019).

[17] CMS Collaboration, Search for low mass vector resonances decaying into quark-antiquark pairs in proton-proton collisions at $\sqrt{s}=13 \mathrm{TeV}$, J. High Energy Phys. 01 (2018) 097.

[18] B. A. Dobrescu and C. Frugiuele, Hidden GeV-Scale Interactions of Quarks, Phys. Rev. Lett. 113, 061801 (2014).

[19] CMS Collaboration, The CMS trigger system, J. Instrum. 12, P01020 (2017).

[20] CMS Collaboration, The CMS experiment at the CERN LHC, J. Instrum. 3, S08004 (2008).

[21] CMS Collaboration, Particle-flow reconstruction and global event description with the CMS detector, J. Instrum. 12, P10003 (2017).

[22] CMS Collaboration, Performance of photon reconstruction and identification with the CMS detector in proton-proton collisions at $\sqrt{s}=8 \mathrm{TeV}$, J. Instrum. 10, P08010 (2015). 
[23] M. Cacciari, G. P. Salam, and G. Soyez, FastJet user manual, Eur. Phys. J. C 72, 1896 (2012).

[24] M. Cacciari, G. P. Salam, and G. Soyez, The anti- $k_{\mathrm{T}}$ clustering algorithm, J. High Energy Phys. 04 (2008) 063.

[25] D. Bertolini, P. Harris, M. Low, and N. Tran, Pileup per particle identification, J. High Energy Phys. 10 (2014) 059.

[26] CMS Collaboration, Determination of jet energy calibration and transverse momentum resolution in CMS, J. Instrum. 6, 11002 (2011).

[27] CMS Collaboration, Identification of heavy-flavour jets with the CMS detector in pp collisions at $13 \mathrm{TeV}$, J. Instrum. 13, P05011 (2018).

[28] B. A. Dobrescu and F. Yu, Coupling-mass mapping of dijet peak searches, Phys. Rev. D 88, 035021 (2013); Erratum, Phys. Rev. D 90, 079901 (2014).

[29] J. Alwall, R. Frederix, S. Frixione, V. Hirschi, F. Maltoni, O. Mattelaer, H.-S. Shao, T. Stelzer, P. Torrielli, and M. Zaro, The automated computation of tree-level and next-toleading order differential cross sections, and their matching to parton shower simulations, J. High Energy Phys. 07 (2014) 079.

[30] J. Alwall, S. Höche, F. Krauss, N. Lavesson, L. Lönnblad, F. Maltoni, M. L. Mangano, M. Moretti, C. G. Papadopoulos, F. Piccinini, S. Schumann, M. Treccani, J. Winter, and M. Worek, Comparative study of various algorithms for the merging of parton showers and matrix elements in hadronic collisions, Eur. Phys. J. C 53, 473 (2008).

[31] P. Nason, A new method for combining NLO QCD with shower Monte Carlo algorithms, J. High Energy Phys. 11 (2004) 040.

[32] S. Frixione, P. Nason, and C. Oleari, Matching NLO QCD computations with parton shower simulations: The POWHEG method, J. High Energy Phys. 11 (2007) 070.

[33] S. Alioli, P. Nason, C. Oleari, and E. Re, A general framework for implementing NLO calculations in shower Monte Carlo programs: The POWHEG BOX, J. High Energy Phys. 06 (2010) 043.

[34] T. Sjöstrand, S. Ask, J. R. Christiansen, R. Corke, N. Desai, P. Ilten, S. Mrenna, S. Prestel, C. O. Rasmussen, and P. Z. Skands, An introduction to PYTHIA 8.2, Comput. Phys. Commun. 191, 159 (2015).

[35] CMS Collaboration, Event generator tunes obtained from underlying event and multiparton scattering measurements, Eur. Phys. J. C 76, 155 (2016).
[36] S. Agostinelli et al. (GEANT4 Collaboration), GEANT4-A simulation toolkit, Nucl. Instrum. Methods Phys. Res., Sect. A 506, 250 (2003).

[37] R. D. Ball et al. (NNPDF Collaboration), Parton distributions for the LHC Run II, J. High Energy Phys. 04 (2015) 040 .

[38] M. Dasgupta, A. Fregoso, S. Marzani, and G. P. Salam, Towards an understanding of jet substructure, J. High Energy Phys. 09 (2013) 029.

[39] A. J. Larkoski, S. Marzani, G. Soyez, and J. Thaler, Soft drop, J. High Energy Phys. 05 (2014) 146.

[40] CMS Collaboration, Measurement of the differential jet cross section as a function of the jet mass in dijet events from proton-proton collisions at $\sqrt{s}=13 \mathrm{TeV}$, J. High Energy Phys. 11 (2018) 113.

[41] A. J. Larkoski, G. P. Salam, and J. Thaler, Energy correlation functions for jet substructure, J. High Energy Phys. 06 (2013) 108.

[42] I. Moult, L. Necib, and J. Thaler, New angles on energy correlation functions, J. High Energy Phys. 12 (2016) 153.

[43] J. Dolen, P. Harris, S. Marzani, S. Rappoccio, and N. Tran, Thinking outside the ROCs: Designing decorrelated taggers (DDT) for jet substructure, J. High Energy Phys. 05 (2016) 156.

[44] R. A. Fisher, On the interpretation of $\chi^{2}$ from contingency tables, and the calculation of $P$, J. R. Stat. 85, 87 (1922).

[45] A. L. Read, Linear interpolation of histograms, Nucl. Instrum. Methods Phys. Res., Sect. A 425, 357 (1999).

[46] CMS Collaboration, CMS luminosity measurements for the 2016 data taking period, CMS Physics Analysis Summary Report No. CMS-PAS-LUM-17-001, 2017, https://cds.cern .ch/record/2257069.

[47] A. L. Read, Presentation of search results: The $\mathrm{CL}_{s}$ technique, J. Phys. G 28, 2693 (2002).

[48] T. Junk, Confidence level computation for combining searches with small statistics, Nucl. Instrum. Methods Phys. Res., Sect. A 434, 435 (1999).

[49] G. Cowan, K. Cranmer, E. Gross, and O. Vitells, Asymptotic formulae for likelihood-based tests of new physics, Eur. Phys. J. C 71, 1554 (2011); Erratum, Eur. Phys. J. C 73, 2501 (2013).

A. M. Sirunyan, ${ }^{1, a}$ A. Tumasyan, ${ }^{1}$ W. Adam, ${ }^{2}$ F. Ambrogi, ${ }^{2}$ T. Bergauer, ${ }^{2}$ J. Brandstetter, ${ }^{2}$ M. Dragicevic, ${ }^{2}$ J. Erö, ${ }^{2}$ A. Escalante Del Valle, ${ }^{2}$ M. Flechl, ${ }^{2}$ R. Frühwirth, ${ }^{2, b}$ M. Jeitler, ${ }^{2, b}$ N. Krammer, ${ }^{2}$ I. Krätschmer, ${ }^{2}$ D. Liko, ${ }^{2}$ T. Madlener, ${ }^{2}$ I. Mikulec, ${ }^{2}$ N. Rad, ${ }^{2}$ J. Schieck, ${ }^{2, b}$ R. Schöfbeck, ${ }^{2}$ M. Spanring, ${ }^{2}$ D. Spitzbart, ${ }^{2}$ W. Waltenberger, ${ }^{2}$ J. Wittmann, ${ }^{2}$ C.-E. Wulz, ${ }^{2, b}$ M. Zarucki, ${ }^{2}$ V. Drugakov, ${ }^{3}$ V. Mossolov, ${ }^{3}$ J. Suarez Gonzalez, ${ }^{3}$ M. R. Darwish, ${ }^{4}$ E. A. De Wolf, ${ }^{4}$ D. Di Croce, ${ }^{4}$ X. Janssen, ${ }^{4}$ J. Lauwers,${ }^{4}$ A. Lelek, ${ }^{4}$ M. Pieters, ${ }^{4}$ H. Van Haevermaet, ${ }^{4}$ P. Van Mechelen, ${ }^{4}$ S. Van Putte, ${ }^{4}$ N. Van Remortel, ${ }^{4}$ F. Blekman, ${ }^{5}$ E. S. Bols, ${ }^{5}$ S. S. Chhibra, ${ }^{5}$ J. D'Hondt, ${ }^{5}$ J. De Clercq, ${ }^{5}$ G. Flouris, ${ }^{5}$ D. Lontkovskyi, ${ }^{5}$ S. Lowette, ${ }^{5}$ I. Marchesini, ${ }^{5}$ S. Moortgat, ${ }^{5}$ L. Moreels, ${ }^{5}$ Q. Python, ${ }^{5}$ K. Skovpen, ${ }^{5}$ S. Tavernier, ${ }^{5}$ W. Van Doninck, ${ }^{5}$ P. Van Mulders, ${ }^{5}$ I. Van Parijs, ${ }^{5}$ D. Beghin, ${ }^{6}$ B. Bilin, ${ }^{6}$ H. Brun, ${ }^{6}$ B. Clerbaux, ${ }^{6}$ G. De Lentdecker, ${ }^{6}$ H. Delannoy, ${ }^{6}$ B. Dorney, ${ }^{6}$ L. Favart, ${ }^{6}$ A. Grebenyuk, ${ }^{6}$ A. K. Kalsi ${ }^{6}$ J. Luetic, ${ }^{6}$ A. Popov, ${ }^{6}$ N. Postiau, ${ }^{6}$ E. Starling, ${ }^{6}$ L. Thomas, ${ }^{6}$ C. Vander Velde, ${ }^{6}$ P. Vanlaer, ${ }^{6}$ D. Vannerom, ${ }^{6}$ Q. Wang, ${ }^{6}$ T. Cornelis, ${ }^{7}$ D. Dobur, ${ }^{7}$ I. Khvastunov, ${ }^{7, c}$ C. Roskas, ${ }^{7}$ D. Trocino, ${ }^{7}$ 
M. Tytgat,${ }^{7}$ W. Verbeke, ${ }^{7}$ B. Vermassen, ${ }^{7}$ M. Vit, ${ }^{7}$ N. Zaganidis, ${ }^{7}$ O. Bondu, ${ }^{8}$ G. Bruno, ${ }^{8}$ C. Caputo, ${ }^{8}$ P. David, ${ }^{8}$ C. Delaere, ${ }^{8}$ M. Delcourt, ${ }^{8}$ A. Giammanco, ${ }^{8}$ G. Krintiras, ${ }^{8}$ V. Lemaitre, ${ }^{8}$ A. Magitteri, ${ }^{8}$ K. Piotrzkowski, ${ }^{8}$ J. Prisciandaro, ${ }^{8}$ A. Saggio, ${ }^{8}$ M. Vidal Marono, ${ }^{8}$ P. Vischia, ${ }^{8}$ J. Zobec ${ }^{8}$ F. L. Alves, ${ }^{9}$ G. A. Alves, ${ }^{9}$ G. Correia Silva, ${ }^{9}$ C. Hensel, ${ }^{9}$ A. Moraes, ${ }^{9}$ P. Rebello Teles,${ }^{9}$ E. Belchior Batista Das Chagas, ${ }^{10}$ W. Carvalho, ${ }^{10}$ J. Chinellato, ${ }^{10, d}$ E. Coelho, ${ }^{10}$ E. M. Da Costa, ${ }^{10}$ G. G. Da Silveira, ${ }^{10, \mathrm{e}}$ D. De Jesus Damiao, ${ }^{10}$ C. De Oliveira Martins, ${ }^{10}$ S. Fonseca De Souza, ${ }^{10}$ L. M. Huertas Guativa, ${ }^{10}$ H. Malbouisson, ${ }^{10}$ J. Martins,${ }^{10}$ D. Matos Figueiredo, ${ }^{10}$ M. Medina Jaime, ${ }^{10, f}$ M. Melo De Almeida, ${ }^{10}$ C. Mora Herrera, ${ }^{10}$ L. Mundim, ${ }^{10}$ H. Nogima, ${ }^{10}$ W. L. Prado Da Silva, ${ }^{10}$ L. J. Sanchez Rosas, ${ }^{10}$ A. Santoro, ${ }^{10}$ A. Sznajder,${ }^{10}$ M. Thiel,${ }^{10}$ E. J. Tonelli Manganote, ${ }^{10, d}$ F. Torres Da Silva De Araujo, ${ }^{10}$ A. Vilela Pereira, ${ }^{10}$ S. Ahuja, ${ }^{11 a}$ C. A. Bernardes, ${ }^{11 a}$ L. Calligaris, ${ }^{11 \mathrm{a}}$ D. De Souza Lemos, ${ }^{11 \mathrm{a}}$ T. R. Fernandez Perez Tomei, ${ }^{11 \mathrm{a}}$ E. M. Gregores, ${ }^{11 \mathrm{a}, 11 \mathrm{~b}}$ P. G. Mercadante, ${ }^{11,11 \mathrm{~b}}$ S. F. Novaes, ${ }^{11 a}$ Sandra S. Padula, ${ }^{11 a}$ A. Aleksandrov, ${ }^{12}$ G. Antchev, ${ }^{12}$ R. Hadjiiska, ${ }^{12}$ P. Iaydjiev, ${ }^{12}$ A. Marinov, ${ }^{12}$ M. Misheva, ${ }^{12}$ M. Rodozov, ${ }^{12}$ M. Shopova, ${ }^{12}$ G. Sultanov, ${ }^{12}$ A. Dimitrov, ${ }^{13}$ L. Litov, ${ }^{13}$ B. Pavlov, ${ }^{13}$ P. Petkov, ${ }^{13}$ W. Fang, ${ }^{14, g}$ X. Gao, ${ }^{14, g}$ L. Yuan,${ }^{14}$ M. Ahmad, ${ }^{15}$ G. M. Chen, ${ }^{15}$ H. S. Chen, ${ }^{15}$ M. Chen,${ }^{15}$ C. H. Jiang, ${ }^{15}$ D. Leggat, ${ }^{15}$ H. Liao, ${ }^{15}$ Z. Liu,${ }^{15}$ S. M. Shaheen, ${ }^{15, h}$ A. Spiezia, ${ }^{15}$ J. Tao, ${ }^{15}$ E. Yazgan, ${ }^{15}$ H. Zhang, ${ }^{15}$ S. Zhang, ${ }^{15, h}$ J. Zhao, ${ }^{15}$ A. Agapitos, ${ }^{16}$ Y. Ban, ${ }^{16}$ G. Chen, ${ }^{16}$ A. Levin, ${ }^{16}$ J. Li, ${ }^{16}$ L. Li, ${ }^{16}$ Q. Li,${ }^{16}$ Y. Mao, ${ }^{16}$ S. J. Qian, ${ }^{16}$ D. Wang, ${ }^{16}$ Y. Wang, ${ }^{17}$ C. Avila,${ }^{18}$ A. Cabrera, ${ }^{18}$ L. F. Chaparro Sierra, ${ }^{18}$ C. Florez, ${ }^{18}$ C. F. González Hernández,${ }^{18}$ M. A. Segura Delgado, ${ }^{18}$ J. D. Ruiz Alvarez, ${ }^{19}$ D. Giljanović, ${ }^{20}$ N. Godinovic, ${ }^{20}$ D. Lelas, ${ }^{20}$ I. Puljak, ${ }^{20}$ T. Sculac, ${ }^{20}$ Z. Antunovic, ${ }^{21}$ M. Kovac, ${ }^{21}$ V. Brigljevic, ${ }^{22}$ S. Ceci, ${ }^{22}$ D. Ferencek, ${ }^{22}$ K. Kadija, ${ }^{22}$ B. Mesic, ${ }^{22}$ M. Roguljic, ${ }^{22}$ A. Starodumov, ${ }^{22, i}$ T. Susa, ${ }^{22}$ M. W. Ather, ${ }^{23}$ A. Attikis, ${ }^{23}$ E. Erodotou, ${ }^{23}$ A. Ioannou, ${ }^{23}$ M. Kolosova, ${ }^{23}$ S. Konstantinou, ${ }^{23}$ G. Mavromanolakis, ${ }^{23}$ J. Mousa, ${ }^{23}$ C. Nicolaou, ${ }^{23}$ F. Ptochos, ${ }^{23}$ P. A. Razis, ${ }^{23}$ H. Rykaczewski, ${ }^{23}$ D. Tsiakkouri, ${ }^{23}$ M. Finger, ${ }^{24, j}$ M. Finger Jr., ${ }^{24, j}$ A. Kveton, ${ }^{24}$ J. Tomsa, ${ }^{24}$ E. Ayala, ${ }^{25}$ E. Carrera Jarrin, ${ }^{26}$ Y. Assran, ${ }^{27, k, 1}$ S. Elgammal, ${ }^{27, k}$ S. Bhowmik, ${ }^{28}$ A. Carvalho Antunes De Oliveira, ${ }^{28}$ R. K. Dewanjee, ${ }^{28}$ K. Ehataht,${ }^{28}$ M. Kadastik, ${ }^{28}$ M. Raidal, ${ }^{28}$ C. Veelken, ${ }^{28}$ P. Eerola, ${ }^{29}$ L. Forthomme, ${ }^{29}$ H. Kirschenmann, ${ }^{29}$ K. Osterberg, ${ }^{29}$ J. Pekkanen, ${ }^{29}$ M. Voutilainen, ${ }^{29}$ F. Garcia, ${ }^{30}$ J. Havukainen,${ }^{30}$ J. K. Heikkilä, ${ }^{30}$ T. Järvinen, ${ }^{30}$ V. Karimäki ${ }^{30}$ R. Kinnunen, ${ }^{30}$ T. Lampén, ${ }^{30}$ K. Lassila-Perini, ${ }^{30}$ S. Laurila, ${ }^{30}$ S. Lehti, ${ }^{30}$ T. Lindén, ${ }^{30}$ P. Luukka, ${ }^{30}$ T. Mäenpää, ${ }^{30}$ H. Siikonen, ${ }^{30}$ E. Tuominen, ${ }^{30}$ J. Tuominiemi, ${ }^{30}$ T. Tuuva, ${ }^{31}$ M. Besancon, ${ }^{32}$ F. Couderc, ${ }^{32}$ M. Dejardin, ${ }^{32}$ D. Denegri, ${ }^{32}$ B. Fabbro, ${ }^{32}$ J. L. Faure, ${ }^{32}$ F. Ferri, ${ }^{32}$ S. Ganjour, ${ }^{32}$ A. Givernaud, ${ }^{32}$ P. Gras,${ }^{32}$ G. Hamel de Monchenault, ${ }^{32}$ P. Jarry, ${ }^{32}$ C. Leloup, ${ }^{32}$ E. Locci, ${ }^{32}$ J. Malcles, ${ }^{32}$ J. Rander, ${ }^{32}$ A. Rosowsky, ${ }^{32}$ M. Ö. Sahin, ${ }^{32}$ A. Savoy-Navarro, ${ }^{32, m}$ M. Titov, ${ }^{32}$ C. Amendola ${ }^{33}$ F. Beaudette, ${ }^{33}$ P. Busson, ${ }^{33}$ C. Charlot, ${ }^{33}$ B. Diab, ${ }^{33}$ R. Granier de Cassagnac, ${ }^{33}$ I. Kucher, ${ }^{33}$ A. Lobanov,${ }^{33}$ C. Martin Perez, ${ }^{33}$ M. Nguyen, ${ }^{33}$ C. Ochando, ${ }^{33}$ P. Paganini, ${ }^{33}$ J. Rembser, ${ }^{33}$ R. Salerno, ${ }^{33}$ J. B. Sauvan,${ }^{33}$ Y. Sirois, ${ }^{33}$ A. Zabi, ${ }^{33}$ A. Zghiche, ${ }^{33}$ J.-L. Agram, ${ }^{34, n}$ J. Andrea, ${ }^{34}$ D. Bloch, ${ }^{34}$ G. Bourgatte, ${ }^{34}$ J.-M. Brom, ${ }^{34}$ E. C. Chabert, ${ }^{34}$ C. Collard, ${ }^{34}$ E. Conte, ${ }^{34, n}$ J.-C. Fontaine, ${ }^{34, n}$ D. Gelé, ${ }^{34}$ U. Goerlach, ${ }^{34}$ M. Jansová, ${ }^{34}$ A.-C. Le Bihan, ${ }^{34}$ N. Tonon, ${ }^{34}$ P. Van Hove, ${ }^{34}$ S. Gadrat, ${ }^{35}$ S. Beauceron, ${ }^{36}$ C. Bernet,${ }^{36}$ G. Boudoul, ${ }^{36}$ C. Camen,${ }^{36}$ N. Chanon, ${ }^{36}$ R. Chierici, ${ }^{36}$ D. Contardo ${ }^{36}$ P. Depasse, ${ }^{36}$ H. El Mamouni, ${ }^{36}$ J. Fay, ${ }^{36}$ S. Gascon, ${ }^{36}$ M. Gouzevitch, ${ }^{36}$ B. Ille, ${ }^{36}$ Sa. Jain, ${ }^{36}$ F. Lagarde, ${ }^{36}$ I. B. Laktineh ${ }^{36}$ H. Lattaud ${ }^{36}$ M. Lethuillier, ${ }^{36}$ L. Mirabito, ${ }^{36}$ S. Perries, ${ }^{36}$ V. Sordini,${ }^{36}$ G. Touquet, ${ }^{36}$ M. Vander Donckt,${ }^{36}$ S. Viret ${ }^{36}$ T. Toriashvili, ${ }^{37,0}$ Z. Tsamalaidze, ${ }^{38, j}$ C. Autermann, ${ }^{39}$ L. Feld, ${ }^{39}$ M. K. Kiesel,${ }^{39}$ K. Klein, ${ }^{39}$ M. Lipinski, ${ }^{39}$ D. Meuser, ${ }^{39}$ A. Pauls, ${ }^{39}$ M. Preuten,${ }^{39}$ M. P. Rauch, ${ }^{39}$ C. Schomakers, ${ }^{39}$ J. Schulz, ${ }^{39}$ M. Teroerde, ${ }^{39}$ B. Wittmer, ${ }^{39}$ A. Albert, ${ }^{40}$ M. Erdmann, ${ }^{40}$ S. Erdweg, ${ }^{40}$ T. Esch, ${ }^{40}$ B. Fischer, ${ }^{40}$ R. Fischer, ${ }^{40}$ S. Ghosh,${ }^{40}$ T. Hebbeker,${ }^{40}$ K. Hoepfner,${ }^{40}$ H. Keller, ${ }^{40}$ L. Mastrolorenzo, ${ }^{40}$ M. Merschmeyer, ${ }^{40}$ A. Meyer, ${ }^{40}$ P. Millet, ${ }^{40}$ G. Mocellin, ${ }^{40}$ S. Mondal,${ }^{40}$ S. Mukherjee,${ }^{40}$ D. Noll, ${ }^{40}$ A. Novak, ${ }^{40}$ T. Pook, ${ }^{40}$ A. Pozdnyakov,${ }^{40}$ T. Quast, ${ }^{40}$ M. Radziej ${ }^{40}$ Y. Rath, ${ }^{40}$ H. Reithler, ${ }^{40}$ M. Rieger, ${ }^{40}$ A. Schmidt, ${ }^{40}$ S. C. Schuler, ${ }^{40}$ A. Sharma, ${ }^{40}$ S. Thüer ${ }^{40}$ S. Wiedenbeck, ${ }^{40}$ G. Flügge, ${ }^{41}$ O. Hlushchenko, ${ }^{41}$ T. Kress, ${ }^{41}$ T. Müller, ${ }^{41}$ A. Nehrkorn, ${ }^{41}$ A. Nowack,${ }^{41}$ C. Pistone, ${ }^{41}$ O. Pooth, ${ }^{41}$ D. Roy,${ }^{41}$ H. Sert, ${ }^{41}$ A. Stahl,,${ }^{41, p}$ M. Aldaya Martin, ${ }^{42}$ C. Asawatangtrakuldee, ${ }^{42}$ P. Asmuss, ${ }^{42}$ I. Babounikau, ${ }^{42} \mathrm{H}$. Bakhshiansohi ${ }^{42} \mathrm{~K}$. Beernaert, ${ }^{42} \mathrm{O}$. Behnke, ${ }^{42} \mathrm{U}$. Behrens,${ }^{42}$ A. Bermúdez Martínez, ${ }^{42}$ D. Bertsche, ${ }^{42}$ A. A. Bin Anuar, ${ }^{42}$ K. Borras, ${ }^{42, q}$ V. Botta, ${ }^{42}$ A. Campbell, ${ }^{42}$ A. Cardini, ${ }^{42}$ P. Connor, ${ }^{42}$ S. Consuegra Rodríguez, ${ }^{42}$ C. Contreras-Campana, ${ }^{42}$ V. Danilov, ${ }^{42}$ A. De Wit, ${ }^{42}$ M. M. Defranchis, ${ }^{42}$ C. Diez Pardos, ${ }^{42}$ D. Domínguez Damiani, ${ }^{42}$ G. Eckerlin, ${ }^{42}$ D. Eckstein, ${ }^{42}$ T. Eichhorn, ${ }^{42}$ A. Elwood, ${ }^{42}$ E. Eren, ${ }^{42}$ E. Gallo, ${ }^{42, r}$ A. Geiser ${ }^{42}$ J. M. Grados Luyando, ${ }^{42}$ A. Grohsjean, ${ }^{42}$ M. Guthoff, ${ }^{42}$ M. Haranko, ${ }^{42}$ A. Harb, ${ }^{42}$ N. Z. Jomhari, ${ }^{42}$ H. Jung, ${ }^{42}$ A. Kasem, ${ }^{42, q}$ M. Kasemann, ${ }^{42}$ J. Keaveney,${ }^{42}$ C. Kleinwort, ${ }^{42}$ J. Knolle, ${ }^{42}$ D. Krücker, ${ }^{42}$ W. Lange,${ }^{42}$ T. Lenz, ${ }^{42}$ J. Leonard, ${ }^{42}$ J. Lidrych, ${ }^{42}$ K. Lipka, ${ }^{42}$ W. Lohmann, ${ }^{42, s}$ R. Mankel, ${ }^{42}$ I.-A. Melzer-Pellmann, ${ }^{42}$ A. B. Meyer, ${ }^{42}$ M. Meyer, ${ }^{42}$ M. Missiroli, ${ }^{42}$ G. Mittag, ${ }^{42}$ J. Mnich, ${ }^{42}$ A. Mussgiller, ${ }^{42}$ V. Myronenko, ${ }^{42}$ D. Pérez Adán, ${ }^{42}$ S. K. Pflitsch, ${ }^{42}$ D. Pitzl, ${ }^{42}$ A. Raspereza, ${ }^{42}$ A. Saibel, ${ }^{42}$ M. Savitskyi ${ }^{42}$ V. Scheurer, ${ }^{42}$ P. Schütze, ${ }^{42}$ C. Schwanenberger, ${ }^{42}$ R. Shevchenko, ${ }^{42}$ A. Singh ${ }^{42}$ 
H. Tholen, ${ }^{42}$ O. Turkot, ${ }^{42}$ A. Vagnerini,${ }^{42}$ M. Van De Klundert, ${ }^{42}$ G. P. Van Onsem, ${ }^{42}$ R. Walsh, ${ }^{42}$ Y. Wen, ${ }^{42}$ K. Wichmann, ${ }^{42}$ C. Wissing, ${ }^{42}$ O. Zenaiev, ${ }^{42}$ R. Zlebcik ${ }^{42}$ R. Aggleton, ${ }^{43}$ S. Bein,${ }^{43}$ L. Benato, ${ }^{43}$ A. Benecke, ${ }^{43}$ V. Blobel, ${ }^{43}$ T. Dreyer, ${ }^{43}$ A. Ebrahimi, ${ }^{43}$ A. Fröhlich, ${ }^{43}$ C. Garbers, ${ }^{43}$ E. Garutti, ${ }^{43}$ D. Gonzalez, ${ }^{43}$ P. Gunnellini, ${ }^{43}$ J. Haller, ${ }^{43}$ A. Hinzmann, ${ }^{43}$ A. Karavdina, ${ }^{43}$ G. Kasieczka, ${ }^{43}$ R. Klanner,${ }^{43}$ R. Kogler, ${ }^{43}$ N. Kovalchuk, ${ }^{43}$ S. Kurz, ${ }^{43}$ V. Kutzner, ${ }^{43}$ J. Lange,${ }^{43}$ T. Lange, ${ }^{43}$ A. Malara, ${ }^{43}$ D. Marconi ${ }^{43}$ J. Multhaup, ${ }^{43}$ M. Niedziela, ${ }^{43}$ C. E. N. Niemeyer, ${ }^{43}$ D. Nowatschin, ${ }^{43}$ A. Perieanu, ${ }^{43}$ A. Reimers, ${ }^{43}$ O. Rieger, ${ }^{43}$ C. Scharf ${ }^{43}$ P. Schleper, ${ }^{43}$ S. Schumann, ${ }^{43}$ J. Schwandt, ${ }^{43}$ J. Sonneveld, ${ }^{43}$ H. Stadie, ${ }^{43}$ G. Steinbrück, ${ }^{43}$ F. M. Stober, ${ }^{43}$ M. Stöver, ${ }^{43}$ B. Vormwald, ${ }^{43}$ I. Zoi, ${ }^{43}$ M. Akbiyik, ${ }^{44}$ C. Barth,${ }^{44}$ M. Baselga ${ }^{44}$ S. Baur ${ }^{44}$ T. Berger, ${ }^{44}$ E. Butz, ${ }^{44}$ R. Caspart, ${ }^{44}$ T. Chwalek, ${ }^{44}$ W. De Boer, ${ }^{44}$ A. Dierlamm, ${ }^{44}$ K. El Morabit, ${ }^{44}$ N. Faltermann, ${ }^{44}$ M. Giffels, ${ }^{44}$ P. Goldenzweig, ${ }^{44}$ M. A. Harrendorf, ${ }^{44}$ F. Hartmann, ${ }^{44, p}$ U. Husemann, ${ }^{44}$ S. Kudella, ${ }^{44}$ S. Mitra, ${ }^{44}$ M. U. Mozer, ${ }^{44}$ Th. Müller, ${ }^{44}$ M. Musich, ${ }^{44}$ A. Nürnberg, ${ }^{44}$ G. Quast ${ }^{44}$ K. Rabbertz, ${ }^{44}$ M. Schröder ${ }^{44}$ I. Shvetsov, ${ }^{44}$ H. J. Simonis, ${ }^{44}$ R. Ulrich, ${ }^{44}$ M. Weber, ${ }^{44}$ C. Wöhrmann, ${ }^{44}$ R. Wolf,${ }^{44}$ G. Anagnostou, ${ }^{45}$ P. Asenov, ${ }^{45}$ G. Daskalakis, ${ }^{45}$ T. Geralis, ${ }^{45}$ A. Kyriakis, ${ }^{45}$ D. Loukas, ${ }^{45}$ G. Paspalaki, ${ }^{45}$ M. Diamantopoulou, ${ }^{46}$ G. Karathanasis, ${ }^{46}$ P. Kontaxakis, ${ }^{46}$ A. Panagiotou, ${ }^{46}$ I. Papavergou, ${ }^{46}$ N. Saoulidou, ${ }^{46}$ A. Stakia, ${ }^{46}$ K. Theofilatos, ${ }^{46}$ K. Vellidis,${ }^{46}$ G. Bakas, ${ }^{47}$ K. Kousouris, ${ }^{47}$ I. Papakrivopoulos, ${ }^{47}$ G. Tsipolitis, ${ }^{47}$ I. Evangelou, ${ }^{48}$ C. Foudas, ${ }^{48}$ P. Gianneios, ${ }^{48}$ P. Katsoulis,${ }^{48}$ P. Kokkas, ${ }^{48}$ S. Mallios,${ }^{48}$ K. Manitara, ${ }^{48}$ N. Manthos, ${ }^{48}$ I. Papadopoulos, ${ }^{48}$ E. Paradas, ${ }^{48}$ J. Strologas, ${ }^{48}$ F. A. Triantis, ${ }^{48}$ D. Tsitsonis,${ }^{48}$ M. Bartók,${ }^{49, t}$ M. Csanad ${ }^{49}$ P. Major ${ }^{49}$ K. Mandal, ${ }^{49}$ A. Mehta, ${ }^{49}$ M. I. Nagy, ${ }^{49}$ G. Pasztor, ${ }^{49}$ O. Surányi, ${ }^{49}$ G. I. Veres, ${ }^{49}$ G. Bencze ${ }^{50}$ C. Hajdu, ${ }^{50}$ D. Horvath,,${ }^{50, u}$ Á. Hunyadi, ${ }^{50}$ F. Sikler, ${ }^{50}$ T. Á. Vámi, ${ }^{50}$ V. Veszpremi, ${ }^{50}$ G. Vesztergombi,${ }^{50, a, v}$ N. Beni, ${ }^{51}$ S. Czellar, ${ }^{51}$ J. Karancsi, ${ }^{51, t}$ A. Makovec, ${ }^{51}$ J. Molnar, ${ }^{51}$ Z. Szillasi, ${ }^{51}$ P. Raics,${ }^{52}$ D. Teyssier, ${ }^{52}$ Z. L. Trocsanyi, ${ }^{52}$ B. Ujvari, ${ }^{52}$ T. F. Csorgo, ${ }^{53}$ W. J. Metzger, ${ }^{53}$ F. Nemes, ${ }^{53}$ T. Novak, ${ }^{53}$ S. Choudhury, ${ }^{54}$ J. R. Komaragiri, ${ }^{54}$ P. C. Tiwari, ${ }^{54}$

S. Bahinipati, ${ }^{55, w}$ C. Kar ${ }^{55}$ P. Mal, ${ }^{55}$ V. K. Muraleedharan Nair Bindhu, ${ }^{55}$ A. Nayak, ${ }^{55, x}$ S. Roy Chowdhury, ${ }^{55}$ D. K. Sahoo, ${ }^{55, w}$ S. K. Swain, ${ }^{55}$ S. Bansal, ${ }^{56}$ S. B. Beri, ${ }^{56}$ V. Bhatnagar, ${ }^{56}$ S. Chauhan, ${ }^{56}$ R. Chawla, ${ }^{56}$ N. Dhingra, ${ }^{56}$ R. Gupta, ${ }^{56}$ A. Kaur, ${ }^{56}$ M. Kaur, ${ }^{56}$ S. Kaur, ${ }^{56}$ P. Kumari, ${ }^{56}$ M. Lohan, ${ }^{56}$ M. Meena, ${ }^{56}$ K. Sandeep ${ }^{56}$ S. Sharma, ${ }^{56}$ J. B. Singh, ${ }^{56}$ A. K. Virdi, ${ }^{56}$ G. Walia, ${ }^{56}$ A. Bhardwaj, ${ }^{57}$ B. C. Choudhary,${ }^{57}$ R. B. Garg, ${ }^{57}$ M. Gola,${ }^{57}$ S. Keshri, ${ }^{57}$ Ashok Kumar, ${ }^{57}$ S. Malhotra, ${ }^{57}$ M. Naimuddin, ${ }^{57}$ P. Priyanka, ${ }^{57}$ K. Ranjan, ${ }^{57}$ Aashaq Shah, ${ }^{57}$ R. Sharma, ${ }^{57}$ R. Bhardwaj, ${ }^{58, y}$ M. Bharti, ${ }^{58, y}$ R. Bhattacharya ${ }^{58}$ S. Bhattacharya ${ }^{58}$ U. Bhawandeep,${ }^{58, y}$ D. Bhowmik, ${ }^{58}$ S. Dey, ${ }^{58}$ S. Dutta, ${ }^{58}$ S. Ghosh, ${ }^{58}$ M. Maity, ${ }^{58, z}$ K. Mondal, ${ }^{58}$ S. Nandan, ${ }^{58}$ A. Purohit,${ }^{58}$ P. K. Rout, ${ }^{58}$ A. Roy, ${ }^{58}$ G. Saha, ${ }^{58}$ S. Sarkar, ${ }^{58}$ T. Sarkar, ${ }^{58, z}$ M. Sharan, ${ }^{58}$ B. Singh, ${ }^{58, y}$ S. Thakur, ${ }^{58, y}$ P. K. Behera, ${ }^{59}$ P. Kalbhor, ${ }^{59}$ A. Muhammad, ${ }^{59}$ P. R. Pujahari, ${ }^{59}$ A. Sharma,${ }^{59}$ A. K. Sikdar, ${ }^{59}$ R. Chudasama ${ }^{60}$ D. Dutta ${ }^{60}$ V. Jha ${ }^{60}$ V. Kumar ${ }^{60}$ D. K. Mishra, ${ }^{60}$ P. K. Netrakanti, ${ }^{60}$ L. M. Pant, ${ }^{60}$ P. Shukla, ${ }^{60}$ T. Aziz, ${ }^{61}$ M. A. Bhat, ${ }^{61}$ S. Dugad, ${ }^{61}$ G. B. Mohanty,${ }^{61}$ N. Sur, ${ }^{61}$ Ravindra Kumar Verma, ${ }^{61}$ S. Banerjee, ${ }^{62}$ S. Bhattacharya ${ }^{62}$ S. Chatterjee, ${ }^{62}$ P. Das, ${ }^{62}$ M. Guchait, ${ }^{62}$ S. Karmakar, ${ }^{62}$ S. Kumar, ${ }^{62}$ G. Majumder, ${ }^{62}$ K. Mazumdar ${ }^{62}$ N. Sahoo, ${ }^{62}$ S. Sawant, ${ }^{62}$ S. Chauhan, ${ }^{63}$ S. Dube, ${ }^{63}$ V. Hegde, ${ }^{63}$ A. Kapoor, ${ }^{63}$ K. Kothekar, ${ }^{63}$ S. Pandey, ${ }^{63}$ A. Rane, ${ }^{63}$ A. Rastogi ${ }^{63}$ S. Sharma, ${ }^{63}$ S. Chenarani, ${ }^{64, \text { aa }}$ E. Eskandari Tadavani ${ }^{64}$ S. M. Etesami, ${ }^{64, \text { aa }}$ M. Khakzad, ${ }^{64}$

M. Mohammadi Najafabadi, ${ }^{64}$ M. Naseri, ${ }^{64}$ F. Rezaei Hosseinabadi ${ }^{64}$ B. Safarzadeh, ${ }^{64, b b}$ M. Felcini,${ }^{65}$ M. Grunewald, ${ }^{65}$ M. Abbrescia, ${ }_{66 a, 66 b}$ C. Calabria, ${ }^{66 a, 66 b}$ A. Colaleo, ${ }^{66 a}$ D. Creanza, ${ }^{66 a, 66 c}$ L. Cristella, ${ }^{66 a, 66 b}$ N. De Filippis, ${ }^{66 a, 66 c}$ M. De Palma ${ }^{66 a, 66 b}$ A. Di Florio, ${ }^{66 a, 66 b}$ L. Fiore ${ }^{66 a}$ A. Gelmi, ${ }^{66 a, 66 b}$ G. Iaselli, ${ }^{66 a, 66 c}$ M. Ince, ${ }^{66 a, 66 b}$ S. Lezki, ${ }^{66 a, 66 b}$ G. Maggi, ${ }^{66 a, 66 c}$ M. Maggi, ${ }^{66 a}$ G. Miniello, ${ }^{66 a, 66 b}$ S. My ${ }^{66 a, 66 b}$ S. Nuzzo, ${ }^{66 a, 66 b}$ A. Pompili, ${ }^{66 a, 66 b}$ G. Pugliese, ${ }^{66 a, 66 c}$ R. Radogna, ${ }^{66 \mathrm{a}}$ A. Ranieri, ${ }^{66 \mathrm{a}}$ G. Selvaggi, ${ }^{66 \mathrm{a}, 66 \mathrm{~b}}$ L. Silvestris, ${ }^{66 \mathrm{a}}$ R. Venditti, ${ }^{66 \mathrm{a}}$ P. Verwilligen, ${ }^{66 \mathrm{a}}$ G. Abbiendi, ${ }^{67 \mathrm{a}}$ C. Battilana, ${ }^{67,67 b}$ D. Bonacorsi, ${ }^{67 a, 67 b}$ L. Borgonovi, ${ }^{67,67 b}$ S. Braibant-Giacomelli, ${ }^{67 a, 67 b}$ R. Campanini, ${ }^{67 a, 67 b}$ P. Capiluppi ${ }^{67 a, 67 b}$ A. Castro, ${ }^{67,67 b}$ F. R. Cavallo, ${ }^{67 a}$ C. Ciocca,${ }^{67 a}$ G. Codispoti, ${ }^{67 a, 67 b}$ M. Cuffiani,${ }^{67 a, 67 b}$ G. M. Dallavalle, ${ }^{67 a}$ F. Fabbri, ${ }^{67 \mathrm{a}}$ A. Fanfani, ${ }^{67 \mathrm{a}, 67 \mathrm{~b}}$ E. Fontanesi, ${ }^{67 \mathrm{a}}$ P. Giacomelli, ${ }^{67 \mathrm{a}}$ C. Grandi, ${ }^{67 \mathrm{a}}$ L. Guiducci, ${ }^{67 \mathrm{a}, 67 \mathrm{~b}}$ F. Iemmi, ${ }^{67 \mathrm{a}, 67 \mathrm{~b}}$ S. Lo Meo, ${ }^{67 a, c c}$ S. Marcellini, ${ }^{67 a}$ G. Masetti, ${ }^{67 a}$ F. L. Navarria, ${ }^{67 a, 67 b}$ A. Perrotta, ${ }^{67 a}$ F. Primavera, ${ }^{67 a, 67 b}$ A. M. Rossi, ${ }^{67 a, 67 b}$ T. Rovelli, ${ }^{67 a, 67 b}$ G. P. Siroli, ${ }^{67 a, 67 b}$ N. Tosi, ${ }^{67 a}$ S. Albergo, ${ }^{68 a, 68 b, d d}$ S. Costa, ${ }^{68,68 b}$ A. Di Mattia, ${ }^{68 a}$ R. Potenza, ${ }^{68 a, 68 b}$ A. Tricomi, ${ }^{68 a, 68 b, d d}$ C. Tuve, ${ }^{68 a, 68 b}$ G. Barbagli, ${ }^{69 a}$ R. Ceccarelli, ${ }^{69 a}$ K. Chatterjee, ${ }^{69 a, 69 b}$ V. Ciulli, ${ }^{69 a, 69 b}$ C. Civinini, ${ }^{69 a}$ R. D’Alessandro, ${ }^{69 a, 69 b}$ E. Focardi, ${ }^{69 a, 69 b}$ G. Latino, ${ }^{69 a}$ P. Lenzi, ${ }^{69,69 b}$ M. Meschini, ${ }^{69 a}$ S. Paoletti, ${ }^{69 a}$ L. Russo, ${ }^{69 a, e e}$ G. Sguazzoni, ${ }^{69 a}$ D. Strom, ${ }^{69 a}$ L. Viliani, ${ }^{69 a}$ L. Benussi ${ }^{70}$ S. Bianco, ${ }^{70}$ F. Fabbri, ${ }^{70}$ D. Piccolo, ${ }^{70}$ M. Bozzo,${ }^{71 a, 71 b}$ F. Ferro, ${ }^{71 a}$ R. Mulargia ${ }^{71 a, 71 b}$ E. Robutti, ${ }^{71 a}$ S. Tosi, ${ }^{71 a, 71 b}$ A. Benaglia, ${ }^{72 a}$ A. Beschi, ${ }^{72 a, 72 b}$ F. Brivio, ${ }^{72 a, 72 b}$ V. Ciriolo, ${ }^{72 a, 72 b, p}$ S. Di Guida, ${ }^{72 a, 72 b, p}$ M. E. Dinardo, ${ }^{72 a, 72 b}$ P. Dini, ${ }^{72 a}$ S. Fiorendi, ${ }^{72 a, 72 b}$ S. Gennai, ${ }^{72 a}$ A. Ghezzi, ${ }^{72 a, 72 b}$ P. Govoni, ${ }^{72 a, 72 b}$

L. Guzzi, ${ }^{72 a, 72 b}$ M. Malberti, ${ }^{72 a}$ S. Malvezzi, ${ }^{72 a}$ D. Menasce, ${ }^{72 a}$ F. Monti ${ }^{72 a, 72 b}$ L. Moroni, ${ }^{72 a}$ G. Ortona, ${ }^{72 a, 72 b}$ M. Paganoni, ${ }^{72 a, 72 b}$ D. Pedrini, ${ }^{72 a}$ S. Ragazzi, ${ }^{72 a, 72 b}$ T. Tabarelli de Fatis, ${ }^{72 a, 72 b}$ D. Zuolo, ${ }^{72 a, 72 b}$ S. Buontempo, ${ }^{73 a}$ 
N. Cavallo, ${ }^{73 a, 73 c}$ A. De Iorio, ${ }^{73 a, 73 b}$ A. Di Crescenzo, ${ }^{73 a, 73 b}$ F. Fabozzi, ${ }^{73 a, 73 c}$ F. Fienga, ${ }^{73 a}$ G. Galati, ${ }^{73 a}$ A. O. M. Iorio, ${ }^{73 a, 73 b}$ L. Lista, ${ }^{73 a, 73 b}$ S. Meola, ${ }^{73 a, 73 d, p}$ P. Paolucci ${ }^{73 a, p}$ B. Rossi ${ }^{73 a}$ C. Sciacca, ${ }^{73 a, 73 b}$ E. Voevodina,${ }^{73 a, 73 b}$ P. Azzi,${ }^{74 a}$ N. Bacchetta, ${ }^{74 a}$ D. Bisello, ${ }^{74,74 b}$ A. Boletti, ${ }^{74,74 b}$ A. Bragagnolo, ${ }^{74 a}$ R. Carlin, ${ }^{74 a, 74 b}$ P. Checchia, ${ }^{74 a}$ M. Dall'Osso, ${ }^{74 a, 74 b}$ P. De Castro Manzano, ${ }^{74 a}$ T. Dorigo, ${ }^{74 a}$ U. Dosselli, ${ }^{74 a}$ F. Gasparini, ${ }^{74 a, 74 b}$ U. Gasparini, ${ }^{74 a, 74 b}$ A. Gozzelino, ${ }^{74 a}$ S. Y. Hoh, ${ }^{74 a}$ P. Lujan, ${ }^{74 a}$ M. Margoni, ${ }^{74 a, 74 b}$ A. T. Meneguzzo, ${ }^{74,74 b}$ J. Pazzini,${ }^{74 a, 74 b}$ M. Presilla,${ }^{74 a, 74 b}$ P. Ronchese,${ }^{74 a, 74 b}$ R. Rossin, ${ }^{74 a, 74 b}$ F. Simonetto, ${ }^{74 a, 74 b}$ A. Tiko, ${ }^{74 a}$ M. Tosi, ${ }^{74 a, 74 b}$ M. Zanetti, ${ }^{74 a, 74 b}$ P. Zotto, ${ }^{74 a, 74 b}$ G. Zumerle, ${ }^{74 a, 74 b}$ A. Braghieri, ${ }^{75 a}$ P. Montagna ${ }^{75 a, 75 b}$ S. P. Ratti, ${ }^{75 a, 75 b}$ V. Re ${ }^{75 a}$ M. Ressegotti,${ }^{75 a, 75 b}$ C. Riccardi, ${ }^{75 a, 75 b}$ P. Salvini, ${ }^{75 a}$ I. Vai,${ }^{75 a, 75 b}$ P. Vitulo, ${ }^{75 a, 75 b}$ M. Biasini, ${ }^{76 a, 76 b}$ G. M. Bilei, ${ }^{76 a}$ C. Cecchi, ${ }^{76 a, 76 b}$ D. Ciangottini, ${ }^{76 a, 76 b}$ L. Fanò,${ }^{76 a, 76 b}$ P. Lariccia, ${ }^{76 a, 76 b}$ R. Leonardi, ${ }^{76 a, 76 b}$ E. Manoni, ${ }^{76 a}$ G. Mantovani, ${ }^{76 a, 76 b}$ V. Mariani, ${ }^{76 a, 76 b}$ M. Menichelli, ${ }^{76 a}$ A. Rossi, ${ }^{76 a, 76 b}$ A. Santocchia, ${ }^{76 a, 76 b}$ D. Spiga ${ }^{76 a}$ K. Androsov, ${ }^{77 a}$ P. Azzurri, ${ }^{77 a}$ G. Bagliesi, ${ }^{77 a}$ V. Bertacchi, ${ }^{77 a, 77 c}$ L. Bianchini, ${ }^{77 a}$ T. Boccali, ${ }^{77 a}$ R. Castaldi, ${ }^{77 a}$ M. A. Ciocci,${ }^{77,77 b}$ R. Dell'Orso, ${ }^{77 a}$ G. Fedi,${ }^{77 a}$ F. Fiori, ${ }^{77 a, 77 c}$ L. Giannini, ${ }^{77,77 c}$ A. Giassi,${ }^{77 a}$ M. T. Grippo, ${ }^{77 a}$ F. Ligabue, ${ }^{77 a, 77 c}$ E. Manca, ${ }^{77,77 c}$ G. Mandorli, ${ }^{77 a, 77 c}$ A. Messineo, ${ }^{77 a, 77 b}$ F. Palla, ${ }^{77 a}$ A. Rizzi, ${ }^{77 a, 77 b}$ G. Rolandi, ${ }^{77 a, f f}$ A. Scribano, ${ }^{77 a}$ P. Spagnolo, ${ }^{77 a}$ R. Tenchini, ${ }^{77 a}$ G. Tonelli, ${ }^{77 a, 77 b}$ N. Turini, ${ }^{77 a}$ A. Venturi, ${ }^{77 a}$ P. G. Verdini, ${ }^{77 a}$ F. Cavallari, ${ }^{78 a}$ M. Cipriani, ${ }^{78 a, 78 b}$ D. Del Re ${ }^{78 a, 78 b}$ E. Di Marco, ${ }^{78 a, 78 b}$ M. Diemoz ${ }^{78 a}$ S. Gelli, ${ }^{78 a, 78 b}$ E. Longo, ${ }^{78 a, 78 b}$ B. Marzocchi, ${ }^{78 a, 78 b}$ P. Meridiani, ${ }^{78 a}$ G. Organtini, ${ }^{78 a, 78 b}$ F. Pandolfi, ${ }^{78 a}$ R. Paramatti, ${ }^{78 a}{ }^{78 b}$ F. Preiato, ${ }^{78 a, 78 b}$ C. Quaranta, ${ }^{78 a, 78 b}$ S. Rahatlou, ${ }^{78 a, 78 b}$ C. Rovelli, ${ }^{78 a}$ F. Santanastasio ${ }^{78 a, 78 b}$ L. Soffi,${ }^{78 a, 78 b}$ N. Amapane, ${ }^{79 a, 79 b}$ R. Arcidiacono, ${ }^{79 a, 79 c}$ S. Argiro, ${ }^{79 a, 79 b}$ M. Arneodo ${ }^{79 a, 79 c}$ N. Bartosik, ${ }^{79 a}$ R. Bellan, ${ }^{79 a, 79 b}$ C. Biino, ${ }^{79 a}$ A. Cappati, ${ }^{79 a, 79 b}$ N. Cartiglia ${ }^{79 a}$ F. Cenna, ${ }^{79 a, 79 b}$

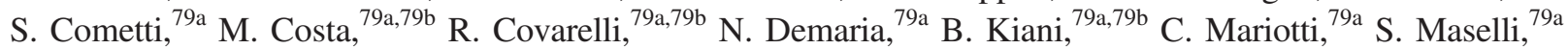
E. Migliore,${ }^{79 a, 79 b}$ V. Monaco,${ }^{79 a, 79 b}$ E. Monteil,${ }^{79 a, 79 b}$ M. Monteno, ${ }^{79 a}$ M. M. Obertino, ${ }^{79 a, 79 b}$ L. Pacher, ${ }^{79 a, 79 b}$ N. Pastrone, ${ }^{79 a}$ M. Pelliccioni, ${ }^{79 a}$ G. L. Pinna Angioni, ${ }^{79 a, 79 b}$ A. Romero, ${ }^{79 a, 79 b}$ M. Ruspa,${ }^{79 a, 79 c}$ R. Sacchi, ${ }^{79 a, 79 b}$ R. Salvatico, ${ }^{79 a, 79 b}$ K. Shchelina, ${ }^{79 a, 79 b}$ V. Sola, ${ }^{79 a}$ A. Solano, ${ }^{79 a, 79 b}$ D. Soldi, ${ }^{79 a, 79 b}$ A. Staiano, ${ }^{79 a}$ S. Belforte, ${ }^{80 a}$ V. Candelise, ${ }^{80 a, 80 b}$ M. Casarsa ${ }^{80 a}$ F. Cossutti, ${ }^{80 a}$ A. Da Rold, ${ }^{80 a, 80 b}$ G. Della Ricca,${ }^{80 a}{ }^{80 b}$ F. Vazzoler ${ }^{80 a, 80 b}$ A. Zanetti, ${ }^{80 a}$ B. Kim, ${ }^{81}$ D. H. Kim, ${ }^{81}$ G. N. Kim ${ }^{81}$ M. S. Kim, ${ }^{81}$ J. Lee ${ }^{81}$ S. W. Lee, ${ }^{81}$ C. S. Moon,${ }^{81}$ Y. D. Oh, ${ }^{81}$ S. I. Pak, ${ }^{81}$ S. Sekmen, ${ }^{81}$ D. C. Son, ${ }^{81}$ Y. C. Yang, ${ }^{81}$ H. Kim, ${ }^{82}$ D. H. Moon, ${ }^{82}$ G. Oh ${ }^{82}$ B. Francois, ${ }^{83}$ T. J. Kim, ${ }^{83}$ J. Park, ${ }^{83}$ S. Cho ${ }^{84}$ S. Choi, ${ }^{84}$ Y. Go, ${ }^{84}$ D. Gyun,${ }^{84}$ S. Ha, ${ }^{84}$ B. Hong ${ }^{84}$ Y. Jo, ${ }^{84}$ K. Lee, ${ }^{84}$ K. S. Lee ${ }^{84}$ S. Lee, ${ }^{84}$ J. Lim, ${ }^{84}$ J. Park, ${ }^{84}$ S. K. Park,${ }^{84}$ Y. Roh, ${ }^{84}$ J. Goh,${ }^{85}$ H. S. Kim,${ }^{86}$ J. Almond, ${ }^{87}$ J. H. Bhyun, ${ }^{87}$ J. Choi,${ }^{87}$ S. Jeon, ${ }^{87}$ J. Kim, ${ }^{87}$ J. S. Kim, ${ }^{87}$ H. Lee, ${ }^{87}$ K. Lee, ${ }^{87}$ S. Lee ${ }^{87}$ K. Nam, ${ }^{87}$ S. B. Oh, ${ }^{87}$ B. C. Radburn-Smith, ${ }^{87}$ S. h. Seo, ${ }^{87}$ U. K. Yang, ${ }^{87}$ H. D. Yoo, ${ }^{87}$ I. Yoon, ${ }^{87}$ G. B. Yu, ${ }^{87}$ D. Jeon, ${ }^{88}$ H. Kim, ${ }^{88}$ J. H. Kim, ${ }^{88}$ J. S. H. Lee, ${ }^{88}$ I. C. Park,${ }^{88}$ I. Watson, ${ }^{88}$ Y. Choi,${ }^{89}$ C. Hwang ${ }^{89}$ Y. Jeong,${ }^{89}$ J. Lee ${ }^{89}$ Y. Lee,${ }^{89}$ I. Yu,${ }^{89}$ V. Veckalns,${ }^{90, g g}$ V. Dudenas, ${ }^{91}$ A. Juodagalvis, ${ }^{91}$ J. Vaitkus, ${ }^{91}$ Z. A. Ibrahim, ${ }^{92}$ F. Mohamad Idris, ${ }^{92, \text { hh }}$ W. A. T. Wan Abdullah, ${ }^{92}$ M. N. Yusli, ${ }^{92}$ Z. Zolkapli, ${ }^{92}$ J. F. Benitez, ${ }^{93}$ A. Castaneda Hernandez, ${ }^{93}$ J. A. Murillo Quijada ${ }^{93}$ L. Valencia Palomo, ${ }^{93}$ H. Castilla-Valdez, ${ }^{94}$ E. De La Cruz-Burelo, ${ }^{94}$ M. C. Duran-Osuna, ${ }^{94}$ I. Heredia-De La Cruz, ${ }^{94, i i}$ R. Lopez-Fernandez, ${ }^{94}$ R. I. Rabadan-Trejo, ${ }^{94}$ G. Ramirez-Sanchez, ${ }^{94}$ R. Reyes-Almanza, ${ }^{94}$ A. Sanchez-Hernandez, ${ }^{94}$ S. Carrillo Moreno, ${ }^{95}$ C. Oropeza Barrera, ${ }^{95}$ M. Ramirez-Garcia,${ }^{95}$ F. Vazquez Valencia, ${ }^{95}$ J. Eysermans,${ }^{96}$ I. Pedraza, ${ }^{96}$ H. A. Salazar Ibarguen, ${ }^{96}$ C. Uribe Estrada, ${ }^{96}$ A. Morelos Pineda,${ }^{97}$ N. Raicevic, ${ }^{98}$ D. Krofcheck,${ }^{99}$ S. Bheesette, ${ }^{100}$ P. H. Butler, ${ }^{100}$ A. Ahmad, ${ }^{101}$ M. Ahmad, ${ }^{101}$ Q. Hassan, ${ }^{101}$ H. R. Hoorani, ${ }^{101}$ W. A. Khan, ${ }^{101}$ M. A. Shah,${ }^{101}$ M. Shoaib, ${ }^{101}$ M. Waqas, ${ }^{101}$ V. Avati, ${ }^{102}$ L. Grzanka, ${ }^{102}$ M. Malawski, ${ }^{102}$ H. Bialkowska, ${ }^{103}$ M. Bluj, ${ }^{103}$ B. Boimska, ${ }^{103}$ M. Górski, ${ }^{103}$ M. Kazana, ${ }^{103}$ M. Szleper, ${ }^{103}$ P. Zalewski, ${ }^{103}$ K. Bunkowski, ${ }^{104}$ A. Byszuk, ${ }^{104, j j}$ K. Doroba, ${ }^{104}$ A. Kalinowski, ${ }^{104}$ M. Konecki, ${ }^{104}$ J. Krolikowski, ${ }^{104}$ M. Misiura, ${ }^{104}$ M. Olszewski, ${ }^{104}$ A. Pyskir, ${ }^{104}$ M. Walczak, ${ }^{104}$ M. Araujo, ${ }^{105}$ P. Bargassa, ${ }^{105}$ D. Bastos, ${ }^{105}$ A. Di Francesco, ${ }^{105}$ P. Faccioli, ${ }^{105}$ B. Galinhas, ${ }^{105}$ M. Gallinaro, ${ }^{105}$ J. Hollar, ${ }^{105}$ N. Leonardo, ${ }^{105}$ J. Seixas, ${ }^{105}$ G. Strong, ${ }^{105}$ O. Toldaiev, ${ }^{105}$ J. Varela, ${ }^{105}$ S. Afanasiev, ${ }^{106}$ P. Bunin, ${ }^{106}$ M. Gavrilenko, ${ }^{106}$ I. Golutvin, ${ }^{106}$ I. Gorbunov, ${ }^{106}$ A. Kamenev, ${ }^{106}$ V. Karjavine, ${ }^{106}$ A. Lanev, ${ }^{106}$ A. Malakhov, ${ }^{106}$ V. Matveev, ${ }^{106, k k, 11}$ P. Moisenz,${ }^{106}$ V. Palichik, ${ }^{106}$ V. Perelygin, ${ }^{106}$ M. Savina, ${ }^{106}$ S. Shmatov, ${ }^{106}$ S. Shulha, ${ }^{106}$ N. Skatchkov, ${ }^{106}$ V. Smirnov, ${ }^{106}$ N. Voytishin,${ }^{106}$ A. Zarubin, ${ }^{106}$ L. Chtchipounov, ${ }^{107}$ V. Golovtsov, ${ }^{107}$ Y. Ivanov, ${ }^{107}$ V. Kim, ${ }^{107, \mathrm{~mm}}$ E. Kuznetsova, ${ }^{107, \mathrm{nn}}$ P. Levchenko, ${ }^{107}$ V. Murzin, ${ }^{107}$ V. Oreshkin, ${ }^{107}$ I. Smirnov, ${ }^{107}$ D. Sosnov, ${ }^{107}$ V. Sulimov, ${ }^{107}$ L. Uvarov,${ }^{107}$ A. Vorobyev ${ }^{107}$ Yu. Andreev, ${ }^{108}$ A. Dermenev, ${ }^{108}$ S. Gninenko, ${ }^{108}$ N. Golubev, ${ }^{108}$ A. Karneyeu, ${ }^{108}$ M. Kirsanov, ${ }^{108}$ N. Krasnikov, ${ }^{108}$ A. Pashenkov, ${ }^{108}$ D. Tlisov, ${ }^{108}$ A. Toropin, ${ }^{108}$ V. Epshteyn, ${ }^{109}$ V. Gavrilov, ${ }^{109}$ N. Lychkovskaya ${ }^{109}$ A. Nikitenko, ${ }^{109,00}$ V. Popov, ${ }^{109}$ I. Pozdnyakov, ${ }^{109}$ G. Safronov, ${ }^{109}$ A. Spiridonov, ${ }^{109}$ A. Stepennov, ${ }^{109}$ M. Toms,${ }^{109}$ E. Vlasov, ${ }^{109}$ A. Zhokin, ${ }^{109}$ T. Aushev, ${ }^{110}$ M. Chadeeva, ${ }^{11, p p}$ S. Polikarpov, ${ }^{111, p p}$ E. Popova, ${ }^{111}$ V. Rusinov, ${ }^{111}$ V. Andreev, ${ }^{112}$ M. Azarkin, ${ }^{112}$ I. Dremin, ${ }^{12,11}$ M. Kirakosyan, ${ }^{112}$ A. Terkulov, ${ }^{112}$ A. Baskakov, ${ }^{113}$ A. Belyaev, ${ }^{113}$ E. Boos, ${ }^{113}$ V. Bunichev, ${ }^{113}$ M. Dubinin, ${ }^{113, q q}$ L. Dudko, ${ }^{113}$ A. Ershov, ${ }^{113}$ 
A. Gribushin, ${ }^{113}$ V. Klyukhin, ${ }^{113}$ O. Kodolova, ${ }^{113}$ I. Lokhtin, ${ }^{113}$ S. Obraztsov, ${ }^{113}$ V. Savrin, ${ }^{113}$ A. Barnyakov, ${ }^{114, \text { rr }}$ V. Blinov, ${ }^{114, \text { rr }}$ T. Dimova, ${ }^{114, \text { rr }}$ L. Kardapoltsev, ${ }^{114, \text { rr }}$ Y. Skovpen, ${ }^{114, \text { rr }}$ I. Azhgirey, ${ }^{115}$ I. Bayshev, ${ }^{115}$ S. Bitioukov, ${ }^{115}$ V. Kachanov, ${ }^{115}$ D. Konstantinov, ${ }^{115}$ P. Mandrik, ${ }^{115}$ V. Petrov, ${ }^{115}$ R. Ryutin, ${ }^{115}$ S. Slabospitskii, ${ }^{115}$ A. Sobol, ${ }^{115}$ S. Troshin, ${ }^{115}$ N. Tyurin, ${ }^{115}$ A. Uzunian, ${ }^{115}$ A. Volkov, ${ }^{115}$ A. Babaev, ${ }^{116}$ A. Iuzhakov, ${ }^{116}$ V. Okhotnikov, ${ }^{116}$ V. Borchsh,${ }^{117}$ V. Ivanchenko, ${ }^{117}$ E. Tcherniaev, ${ }^{117}$ P. Adzic, ${ }^{118, s s}$ P. Cirkovic, ${ }^{118}$ D. Devetak, ${ }^{118}$ M. Dordevic, ${ }^{118}$ P. Milenovic, ${ }^{118, t t}$ J. Milosevic, ${ }^{118}$ M. Stojanovic, ${ }^{118}$ M. Aguilar-Benitez, ${ }^{119}$ J. Alcaraz Maestre, ${ }^{119}$ A. Álvarez Fernández, ${ }^{119}$ I. Bachiller, ${ }^{119}$ M. Barrio Luna, ${ }^{119}$ J. A. Brochero Cifuentes, ${ }^{119}$ C. A. Carrillo Montoya, ${ }^{119}$ M. Cepeda, ${ }^{119}$ M. Cerrada, ${ }^{119}$ N. Colino, ${ }^{119}$ B. De La Cruz, ${ }^{119}$ A. Delgado Peris, ${ }^{119}$ C. Fernandez Bedoya, ${ }^{119}$ J. P. Fernández Ramos, ${ }^{119}$ J. Flix, ${ }^{119}$ M. C. Fouz, ${ }^{119}$ O. Gonzalez Lopez, ${ }^{119}$ S. Goy Lopez, ${ }^{119}$ J. M. Hernandez ${ }^{119}$ M. I. Josa ${ }^{119}$ D. Moran, ${ }^{119}$ Á. Navarro Tobar, ${ }^{119}$ A. Pérez-Calero Yzquierdo, ${ }^{119}$ J. Puerta Pelayo, ${ }^{119}$ I. Redondo, ${ }^{119}$ L. Romero, ${ }^{119}$ S. Sánchez Navas, ${ }^{119}$ M. S. Soares, ${ }^{119}$ A. Triossi, ${ }^{119}$ C. Willmott,,${ }^{119}$ C. Albajar, ${ }^{120}$ J. F. de Trocóniz, ${ }^{120}$ J. Cuevas, ${ }^{121}$ C. Erice, ${ }^{121}$ J. Fernandez Menendez, ${ }^{121}$ S. Folgueras, ${ }^{121}$

I. Gonzalez Caballero, ${ }^{121}$ J. R. González Fernández, ${ }^{121}$ E. Palencia Cortezon, ${ }^{121}$ V. Rodríguez Bouza, ${ }^{121}$ S. Sanchez Cruz, ${ }^{121}$ J. M. Vizan Garcia, ${ }^{121}$ I. J. Cabrillo, ${ }^{122}$ A. Calderon, ${ }^{122}$ B. Chazin Quero, ${ }^{122}$ J. Duarte Campderros, ${ }^{122}$ M. Fernandez, ${ }^{122}$ P. J. Fernández Manteca, ${ }^{122}$ A. García Alonso, ${ }^{122}$ G. Gomez, ${ }^{122}$ C. Martinez Rivero, ${ }^{122}$ P. Martinez Ruiz del Arbol, ${ }^{122}$ F. Matorras, ${ }^{122}$ J. Piedra Gomez, ${ }^{122}$ C. Prieels, ${ }^{122}$ T. Rodrigo, ${ }^{122}$ A. Ruiz-Jimeno, ${ }^{122}$ L. Scodellaro, ${ }^{122}$ N. Trevisani, ${ }^{122}$ I. Vila, ${ }^{122}$ K. Malagalage, ${ }^{123}$ W. G. D. Dharmaratna, ${ }^{124}$ N. Wickramage, ${ }^{124}$ D. Abbaneo, ${ }^{125}$ B. Akgun, ${ }^{125}$ E. Auffray, ${ }^{125}$ G. Auzinger, ${ }^{125}$ J. Baechler, ${ }^{125}$ P. Baillon, ${ }^{125}$ A. H. Ball, ${ }^{125}$ D. Barney, ${ }^{125}$ J. Bendavid, ${ }^{125}$ M. Bianco, ${ }^{125}$ A. Bocci, ${ }^{125}$ E. Bossini, ${ }^{125}$ C. Botta, ${ }^{125}$ E. Brondolin, ${ }^{125}$ T. Camporesi, ${ }^{125}$ A. Caratelli, ${ }^{125}$ G. Cerminara, ${ }^{125}$ E. Chapon, ${ }^{125}$ G. Cucciati, ${ }^{125}$ D. d'Enterria, ${ }^{125}$ A. Dabrowski, ${ }^{125}$ N. Daci, ${ }^{125}$ V. Daponte, ${ }^{125}$ A. David, ${ }^{125}$ A. De Roeck, ${ }^{125}$ N. Deelen, ${ }^{125}$ M. Deile, ${ }^{125}$ M. Dobson, ${ }^{125}$ M. Dünser, ${ }^{125}$ N. Dupont, ${ }^{125}$ A. Elliott-Peisert, ${ }^{125}$ F. Fallavollita, ${ }^{125, \text { uu }}$ D. Fasanella, ${ }^{125}$ G. Franzoni, ${ }^{125}$ J. Fulcher, ${ }^{125}$ W. Funk, ${ }^{125}$ S. Giani, ${ }^{125}$ D. Gigi, ${ }^{125}$ A. Gilbert, ${ }^{125}$ K. Gill, ${ }^{125}$ F. Glege, ${ }^{125}$ M. Gruchala, ${ }^{125}$ M. Guilbaud, ${ }^{125}$ D. Gulhan, ${ }^{125}$ J. Hegeman, ${ }^{125}$ C. Heidegger, ${ }^{125}$ Y. Iiyama, ${ }^{125}$ V. Innocente, ${ }^{125}$ A. Jafari, ${ }^{125}$ P. Janot,${ }^{125}$ O. Karacheban, ${ }^{125, s}$ J. Kaspar, ${ }^{125}$ J. Kieseler, ${ }^{125}$ M. Krammer, ${ }^{125, b}$ C. Lange, ${ }^{125}$ P. Lecoq,${ }^{125}$ C. Lourenço, ${ }^{125}$ L. Malgeri, ${ }^{125}$ M. Mannelli, ${ }^{125}$ A. Massironi, ${ }^{125}$ F. Meijers, ${ }^{125}$ J. A. Merlin, ${ }^{125}$ S. Mersi ${ }^{125}$ E. Meschi, ${ }^{125}$ F. Moortgat, ${ }^{125}$ M. Mulders, ${ }^{125}$ J. Ngadiuba, ${ }^{125}$ S. Nourbakhsh, ${ }^{125}$ S. Orfanelli, ${ }^{125}$ L. Orsini,${ }^{125}$ F. Pantaleo, ${ }^{125, p}$ L. Pape, ${ }^{125}$ E. Perez, ${ }^{125}$ M. Peruzzi, ${ }^{125}$ A. Petrilli, ${ }^{125}$ G. Petrucciani, ${ }^{125}$ A. Pfeiffer, ${ }^{125}$ M. Pierini ${ }^{125}$ F. M. Pitters, ${ }^{125}$ M. Quinto, ${ }^{125}$ D. Rabady, ${ }^{125}$ A. Racz, ${ }^{125}$ M. Rovere, ${ }^{125}$ H. Sakulin, ${ }^{125}$ C. Schäfer, ${ }^{125}$ C. Schwick, ${ }^{125}$ M. Selvaggi, ${ }^{125}$ A. Sharma, ${ }^{125}$ P. Silva, ${ }^{125}$ W. Snoeys, ${ }^{125}$ P. Sphicas, ${ }^{125, v v}$ J. Steggemann, ${ }^{125}$ V. R. Tavolaro, ${ }^{125}$ D. Treille, ${ }^{125}$ A. Tsirou, ${ }^{125}$ A. Vartak, ${ }^{125}$ M. Verzetti, ${ }^{125}$ W. D. Zeuner, ${ }^{125}$

L. Caminada, ${ }^{126, w w}$ K. Deiters, ${ }^{126}$ W. Erdmann, ${ }^{126}$ R. Horisberger,${ }^{126}$ Q. Ingram,${ }^{126}$ H. C. Kaestli, ${ }^{126}$ D. Kotlinski, ${ }^{126}$

U. Langenegger, ${ }^{126}$ T. Rohe, ${ }^{126}$ S. A. Wiederkehr, ${ }^{126}$ M. Backhaus, ${ }^{127}$ P. Berger, ${ }^{127}$ N. Chernyavskaya, ${ }^{127}$ G. Dissertori, ${ }^{127}$ M. Dittmar, ${ }^{127}$ M. Donegà, ${ }^{127}$ C. Dorfer, ${ }^{127}$ T. A. Gómez Espinosa, ${ }^{127}$ C. Grab ${ }^{127}$ D. Hits, ${ }^{127}$ T. Klijnsma, ${ }^{127}$ W. Lustermann, ${ }^{127}$ R. A. Manzoni, ${ }^{127}$ M. Marionneau, ${ }^{127}$ M. T. Meinhard, ${ }^{127}$ F. Micheli, ${ }^{127}$ P. Musella, ${ }^{127}$

F. Nessi-Tedaldi, ${ }^{127}$ F. Pauss, ${ }^{127}$ G. Perrin, ${ }^{127}$ L. Perrozzi, ${ }^{127}$ S. Pigazzini, ${ }^{127}$ M. Reichmann, ${ }^{127}$ C. Reissel, ${ }^{127}$ T. Reitenspiess, ${ }^{127}$ D. Ruini, ${ }^{127}$ D. A. Sanz Becerra,${ }^{127}$ M. Schönenberger, ${ }^{127}$ L. Shchutska, ${ }^{127}$ M. L. Vesterbacka Olsson, ${ }^{127}$ R. Wallny, ${ }^{127}$ D. H. Zhu, ${ }^{127}$ T. K. Aarrestad, ${ }^{128}$ C. Amsler, ${ }^{128, x x}$ D. Brzhechko, ${ }^{128}$ M. F. Canelli, ${ }^{128}$ A. De Cosa, ${ }^{128}$ R. Del Burgo, ${ }^{128}$ S. Donato, ${ }^{128}$ C. Galloni, ${ }^{128}$ B. Kilminster, ${ }^{128}$ S. Leontsinis, ${ }^{128}$ V. M. Mikuni, ${ }^{128}$ I. Neutelings, ${ }^{128}$ G. Rauco, ${ }^{128}$ P. Robmann, ${ }^{128}$ D. Salerno, ${ }^{128}$ K. Schweiger, ${ }^{128}$ C. Seitz, ${ }^{128}$ Y. Takahashi, ${ }^{128}$ S. Wertz, ${ }^{128}$ A. Zucchetta, ${ }^{128}$ T. H. Doan, ${ }^{129}$ C. M. Kuo, ${ }^{129}$ W. Lin, ${ }^{129}$ S. S. Yu, ${ }^{129}$ P. Chang, ${ }^{130}$ Y. Chao, ${ }^{130}$ K. F. Chen, ${ }^{130}$ P. H. Chen, ${ }^{130}$ W.-S. Hou, ${ }^{130}$ Y. y. Li, ${ }^{130}$ R.-S. Lu, ${ }^{130}$ E. Paganis, ${ }^{130}$ A. Psallidas, ${ }^{130}$ A. Steen, ${ }^{130}$ B. Asavapibhop, ${ }^{131}$ N. Srimanobhas, ${ }^{131}$ N. Suwonjandee, ${ }^{131}$ A. Bat, ${ }^{132}$ F. Boran, ${ }^{132}$ S. Cerci, ${ }^{132, y y}$ S. Damarseckin, ${ }^{132, z z}$ Z. S. Demiroglu, ${ }^{132}$ F. Dolek, ${ }^{132}$ C. Dozen, ${ }^{132}$ I. Dumanoglu, ${ }^{132}$ G. Gokbulut, ${ }^{132}$ Emine Gurpinar Guler, ${ }^{132, \text { aaa }}$ Y. Guler, ${ }^{132}$ I. Hos, ${ }^{132, \text { bbb }}$ C. Isik, ${ }^{132}$ E. E. Kangal, ${ }^{132, c c c}$ O. Kara, ${ }^{132}$ A. Kayis Topaksu, ${ }^{132}$ U. Kiminsu, ${ }^{132}$ M. Oglakci, ${ }^{132}$ G. Onengut, ${ }^{132}$ K. Ozdemir, ${ }^{132, d d d}$ S. Ozturk, ${ }^{132, \text { eee }}$ A. E. Simsek ${ }^{132}$ D. Sunar Cerci, ${ }^{132, y y}$ U. G. Tok, ${ }^{132}$ S. Turkcapar, ${ }^{132}$ I. S. Zorbakir, ${ }^{132}$ C. Zorbilmez, ${ }^{132}$ B. Isildak, ${ }^{133, f f f}$ G. Karapinar, ${ }^{133, g g g}$ M. Yalvac, ${ }^{133}$ I. O. Atakisi,${ }^{134}$ E. Gülmez, ${ }^{134}$ M. Kaya, ${ }^{134, \text { hhh }}$ O. Kaya, ${ }^{134, \text { iii }}$ B. Kaynak, ${ }^{134}$ Ö. Özçelik, ${ }^{134}$ S. Ozkorucuklu, ${ }^{134, j j j}$ S. Tekten, ${ }^{134}$ E. A. Yetkin, ${ }^{134, k k k}$ A. Cakir, ${ }^{135}$ Y. Komurcu, ${ }^{135}$ S. Sen, ${ }^{135,111}$ B. Grynyov, ${ }^{136}$ L. Levchuk, ${ }^{137}$ F. Ball, ${ }^{138}$ E. Bhal, ${ }^{138}$ S. Bologna, ${ }^{138}$ J. J. Brooke, ${ }^{138}$ D. Burns, ${ }^{138}$ E. Clement, ${ }^{138}$ D. Cussans, ${ }^{138}$ O. Davignon, ${ }^{138}$ H. Flacher, ${ }^{138}$ J. Goldstein, ${ }^{138}$ G. P. Heath ${ }^{138}$ H. F. Heath, ${ }^{138}$ L. Kreczko, ${ }^{138}$ S. Paramesvaran, ${ }^{138}$ B. Penning, ${ }^{138}$ T. Sakuma, ${ }^{138}$ S. Seif El Nasr-Storey ${ }^{138}$ D. Smith, ${ }^{138}$ V. J. Smith, ${ }^{138}$ J. Taylor, ${ }^{138}$ A. Titterton, ${ }^{138}$ K. W. Bell,,${ }^{139}$ A. Belyaev, ${ }^{139, \mathrm{mmm}}$ C. Brew, ${ }^{139}$ R. M. Brown, ${ }^{139}$ D. Cieri, ${ }^{139}$ D. J. A. Cockerill, ${ }^{139}$ J. A. Coughlan, ${ }^{139}$ K. Harder, ${ }^{139}$ 
S. Harper ${ }^{139}$ J. Linacre, ${ }^{139}$ K. Manolopoulos,${ }^{139}$ D. M. Newbold, ${ }^{139}$ E. Olaiya,${ }^{139}$ D. Petyt, ${ }^{139}$ T. Reis, ${ }^{139}$ T. Schuh, ${ }^{139}$ C. H. Shepherd-Themistocleous, ${ }^{139}$ A. Thea, ${ }^{139}$ I. R. Tomalin, ${ }^{139}$ T. Williams, ${ }^{139}$ W. J. Womersley, ${ }^{139}$ R. Bainbridge, ${ }^{140}$ P. Bloch, ${ }^{140}$ J. Borg, ${ }^{140}$ S. Breeze, ${ }^{140}$ O. Buchmuller, ${ }^{140}$ A. Bundock, ${ }^{140}$ Gurpreet Singh Chahal, ${ }^{140, \text { nnn }}$ D. Colling, ${ }^{140}$ P. Dauncey, ${ }^{140}$ G. Davies, ${ }^{140}$ M. Della Negra ${ }^{140}$ R. Di Maria ${ }^{140}$ P. Everaerts, ${ }^{140}$ G. Hall,,${ }^{140}$ G. Iles, ${ }^{140}$ T. James, ${ }^{140}$ M. Komm, ${ }^{140}$ C. Laner, ${ }^{140}$ L. Lyons, ${ }^{140}$ A.-M. Magnan, ${ }^{140}$ S. Malik, ${ }^{140}$ A. Martelli, ${ }^{140}$ V. Milosevic, ${ }^{140}$ J. Nash, ${ }^{140,000}$ V. Palladino, ${ }^{140}$ M. Pesaresi, ${ }^{140}$ D. M. Raymond, ${ }^{140}$ A. Richards, ${ }^{140}$ A. Rose,${ }^{140}$ E. Scott, ${ }^{140}$ C. Seez, ${ }^{140}$ A. Shtipliyski, ${ }^{140}$ M. Stoye, ${ }^{140}$ T. Strebler, ${ }^{140}$ S. Summers, ${ }^{140}$ A. Tapper, ${ }^{140}$ K. Uchida, ${ }^{140}$ T. Virdee, ${ }^{140, p}$ N. Wardle, ${ }^{140}$ D. Winterbottom, ${ }^{140}$ J. Wright, ${ }^{140}$ A. G. Zecchinelli, ${ }^{140}$ S. C. Zenz, ${ }^{140}$ J. E. Cole,${ }^{141}$ P. R. Hobson, ${ }^{141}$ A. Khan,${ }^{141}$ P. Kyberd, ${ }^{141}$ C. K. Mackay, ${ }^{141}$ A. Morton, ${ }^{141}$ I. D. Reid, ${ }^{141}$ L. Teodorescu, ${ }^{141}$ S. Zahid ${ }^{141}$ K. Call, ${ }^{142}$ J. Dittmann, ${ }^{142}$ K. Hatakeyama, ${ }^{142}$ C. Madrid, ${ }^{142}$ B. McMaster, ${ }^{142}$ N. Pastika, ${ }^{142}$ C. Smith,${ }^{142}$ R. Bartek, ${ }^{143}$ A. Dominguez, ${ }^{143}$ R. Uniyal, ${ }^{143}$ A. Buccilli, ${ }^{144}$ S. I. Cooper, ${ }^{144}$ C. Henderson, ${ }^{144}$ P. Rumerio, ${ }^{144}$ C. West,${ }^{144}$ D. Arcaro, ${ }^{145}$ T. Bose, ${ }^{145}$ Z. Demiragli, ${ }^{145}$ D. Gastler, ${ }^{145}$ S. Girgis, ${ }^{145}$ D. Pinna,${ }^{145}$ C. Richardson, ${ }^{145}$ J. Rohlf, ${ }^{145}$ D. Sperka, ${ }^{145}$ I. Suarez, ${ }^{145}$ L. Sulak,${ }^{145}$ D. Zou, ${ }^{145}$ G. Benelli, ${ }^{146}$ B. Burkle, ${ }^{146}$ X. Coubez, ${ }^{146}$ D. Cutts, ${ }^{146}$ M. Hadley, ${ }^{146}$ J. Hakala, ${ }^{146}$ U. Heintz, ${ }^{146}$ J. M. Hogan, ${ }^{146, p p p}$ K. H. M. Kwok, ${ }^{146}$ E. Laird ${ }^{146}$ G. Landsberg, ${ }^{146}$ J. Lee, ${ }^{146}$ Z. Mao, ${ }^{146}$ M. Narain, ${ }^{146}$ S. Sagir, ${ }^{146, q q 9}$ R. Syarif, ${ }^{146}$ E. Usai, ${ }^{146}$ D. Yu, ${ }^{146}$ R. Band, ${ }^{147}$

C. Brainerd, ${ }^{147}$ R. Breedon, ${ }^{147}$ M. Calderon De La Barca Sanchez, ${ }^{147}$ M. Chertok, ${ }^{147}$ J. Conway, ${ }^{147}$ R. Conway, ${ }^{147}$

P. T. Cox,${ }^{147}$ R. Erbacher, ${ }^{147}$ C. Flores,${ }^{147}$ G. Funk,${ }^{147}$ F. Jensen, ${ }^{147}$ W. Ko, ${ }^{147}$ O. Kukral, ${ }^{147}$ R. Lander, ${ }^{147}$ M. Mulhearn, ${ }^{147}$

D. Pellett, ${ }^{147}$ J. Pilot, ${ }^{147}$ M. Shi,${ }^{147}$ D. Stolp, ${ }^{147}$ D. Taylor,${ }^{147}$ K. Tos, ${ }^{147}$ M. Tripathi, ${ }^{147}$ Z. Wang, ${ }^{147}$ F. Zhang, ${ }^{147}$ M. Bachtis, ${ }^{148}$ C. Bravo, ${ }^{148}$ R. Cousins, ${ }^{148}$ A. Dasgupta ${ }^{148}$ A. Florent, ${ }^{148}$ J. Hauser, ${ }^{148}$ M. Ignatenko, ${ }^{148}$ N. Mccoll, ${ }^{148}$ S. Regnard, ${ }^{148}$ D. Saltzberg, ${ }^{148}$ C. Schnaible, ${ }^{148}$ V. Valuev, ${ }^{148}$ K. Burt, ${ }^{149}$ R. Clare, ${ }^{149}$ J. W. Gary, ${ }^{149}$

S. M. A. Ghiasi Shirazi, ${ }^{149}$ G. Hanson, ${ }^{149}$ G. Karapostoli,${ }^{149}$ E. Kennedy, ${ }^{149}$ O. R. Long, ${ }^{149}$ M. Olmedo Negrete, ${ }^{149}$ M. I. Paneva, ${ }^{149}$ W. Si, ${ }^{149}$ L. Wang, ${ }^{149}$ H. Wei, ${ }^{149}$ S. Wimpenny, ${ }^{149}$ B. R. Yates, ${ }^{149}$ Y. Zhang, ${ }^{149}$ J. G. Branson, ${ }^{150}$ P. Chang, ${ }^{150}$ S. Cittolin, ${ }^{150}$ M. Derdzinski, ${ }^{150}$ R. Gerosa, ${ }^{150}$ D. Gilbert, ${ }^{150}$ B. Hashemi, ${ }^{150}$ D. Klein, ${ }^{150}$ V. Krutelyov, ${ }^{150}$ J. Letts, ${ }^{150}$ M. Masciovecchio, ${ }^{150}$ S. May, ${ }^{150}$ S. Padhi, ${ }^{150}$ M. Pieri, ${ }^{150}$ V. Sharma, ${ }^{150}$ M. Tadel,${ }^{150}$ F. Würthwein, ${ }^{150}$ A. Yagil, ${ }^{150}$ G. Zevi Della Porta ${ }^{150}$ N. Amin, ${ }^{151}$ R. Bhandari, ${ }^{151}$ C. Campagnari ${ }^{151}$ M. Citron, ${ }^{151}$ V. Dutta, ${ }^{151}$ M. Franco Sevilla, ${ }^{151}$ L. Gouskos, ${ }^{151}$ J. Incandela, ${ }^{151}$ B. Marsh,${ }^{151}$ H. Mei, ${ }^{151}$ A. Ovcharova ${ }^{151}$ H. Qu, ${ }^{151}$ J. Richman, ${ }^{151}$ U. Sarica, ${ }^{151}$ D. Stuart, ${ }^{151}$ S. Wang, ${ }^{151}$ J. Yoo, ${ }^{151}$ D. Anderson, ${ }^{152}$ A. Bornheim, ${ }^{152}$ J. M. Lawhorn, ${ }^{152}$ N. Lu, ${ }^{152}$ H. B. Newman, ${ }^{152}$ T. Q. Nguyen, ${ }^{152}$ J. Pata, ${ }^{152}$ M. Spiropulu, ${ }^{152}$ J. R. Vlimant, ${ }^{152}$ S. Xie, ${ }^{152}$ Z. Zhang, ${ }^{152}$ R. Y. Zhu, ${ }^{152}$ M. B. Andrews, ${ }^{153}$ T. Ferguson, ${ }^{153}$ T. Mudholkar, ${ }^{153}$ M. Paulini, ${ }^{153}$ M. Sun ${ }^{153}$ I. Vorobiev, ${ }^{153}$ M. Weinberg, ${ }^{153}$ J. P. Cumalat, ${ }^{154}$ W. T. Ford, ${ }^{154}$ A. Johnson, ${ }^{154}$ E. MacDonald, ${ }^{154}$ T. Mulholland, ${ }^{154}$ R. Patel, ${ }^{154}$ A. Perloff, ${ }^{154}$ K. Stenson, ${ }^{154}$ K. A. Ulmer, ${ }^{154}$ S. R. Wagner, ${ }^{154}$ J. Alexander, ${ }^{155}$ J. Chaves, ${ }^{155}$ Y. Cheng, ${ }^{155}$ J. Chu, ${ }^{155}$ A. Datta, ${ }^{155}$ A. Frankenthal, ${ }^{155}$ K. Mcdermott,${ }^{155}$ N. Mirman, ${ }^{155}$

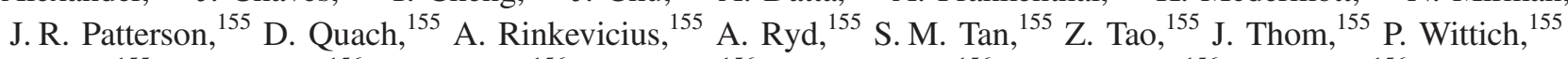
M. Zientek, ${ }^{155}$ S. Abdullin, ${ }^{156}$ M. Albrow, ${ }^{156}$ M. Alyari, ${ }^{156}$ G. Apollinari, ${ }^{156}$ A. Apresyan, ${ }^{156}$ A. Apyan, ${ }^{156}$ S. Banerjee,,${ }^{156}$

L. A. T. Bauerdick, ${ }^{156}$ A. Beretvas, ${ }^{156}$ J. Berryhill ${ }^{156}$ P. C. Bhat, ${ }^{156}$ K. Burkett,${ }^{156}$ J. N. Butler,${ }^{156}$ A. Canepa, ${ }^{156}$

G. B. Cerati, ${ }^{156}$ H. W. K. Cheung, ${ }^{156}$ F. Chlebana, ${ }^{156}$ M. Cremonesi, ${ }^{156}$ J. Duarte, ${ }^{156}$ V. D. Elvira, ${ }^{156}$ J. Freeman, ${ }^{156}$ Z. Gecse ${ }^{156}$ E. Gottschalk, ${ }^{156}$ L. Gray, ${ }^{156}$ D. Green, ${ }^{156}$ S. Grünendahl, ${ }^{156}$ O. Gutsche, ${ }^{156}$ Allison Reinsvold Hall, ${ }^{156}$ J. Hanlon, ${ }^{156}$ R. M. Harris, ${ }^{156}$ S. Hasegawa, ${ }^{156}$ R. Heller, ${ }^{156}$ J. Hirschauer, ${ }^{156}$ Z. Hu, ${ }^{156}$ B. Jayatilaka, ${ }^{156}$ S. Jindariani, ${ }^{156}$ M. Johnson, ${ }^{156}$ U. Joshi, ${ }^{156}$ B. Klima, ${ }^{156}$ M. J. Kortelainen, ${ }^{156}$ B. Kreis, ${ }^{156}$ S. Lammel, ${ }^{156}$ J. Lewis, ${ }^{156}$ D. Lincoln, ${ }^{156}$ R. Lipton, ${ }^{156}$ M. Liu, ${ }^{156}$ T. Liu, ${ }^{156}$ J. Lykken, ${ }^{156}$ K. Maeshima ${ }^{156}$ J. M. Marraffino, ${ }^{156}$ D. Mason, ${ }^{156}$ P. McBride,${ }^{156}$ P. Merkel, ${ }^{156}$ S. Mrenna, ${ }^{156}$ S. Nahn, ${ }^{156}$ V. O’Dell ${ }^{156}$ V. Papadimitriou, ${ }^{156}$ K. Pedro, ${ }^{156}$ C. Pena,${ }^{156}$ G. Rakness, ${ }^{156}$ F. Ravera, ${ }^{156}$ L. Ristori, ${ }^{156}$ B. Schneider, ${ }^{156}$ E. Sexton-Kennedy, ${ }^{156}$ N. Smith, ${ }^{156}$ A. Soha, ${ }^{156}$ W. J. Spalding, ${ }^{156}$ L. Spiegel,,${ }^{156}$ S. Stoynev, ${ }^{156}$ J. Strait, ${ }^{156}$ N. Strobbe, ${ }^{156}$ L. Taylor, ${ }^{156}$ S. Tkaczyk, ${ }^{156}$ N. V. Tran, ${ }^{156}$ L. Uplegger, ${ }^{156}$ E. W. Vaandering, ${ }^{156}$ C. Vernieri, ${ }^{156}$ M. Verzocchi, ${ }^{156}$ R. Vidal, ${ }^{156}$ M. Wang, ${ }^{156}$ H. A. Weber, ${ }^{156}$ D. Acosta, ${ }^{157}$ P. Avery, ${ }^{157}$ P. Bortignon, ${ }^{157}$ D. Bourilkov, ${ }^{157}$ A. Brinkerhoff, ${ }^{157}$ L. Cadamuro, ${ }^{157}$ A. Carnes, ${ }^{157}$ V. Cherepanov, ${ }^{157}$ D. Curry, ${ }^{157}$ F. Errico, ${ }^{157}$ R. D. Field, ${ }^{157}$ S. V. Gleyzer, ${ }^{157}$ B. M. Joshi, ${ }^{157}$ M. Kim, ${ }^{157}$ J. Konigsberg, ${ }^{157}$ A. Korytov,${ }^{157}$ K. H. Lo ${ }^{157}$ P. Ma, ${ }^{157}$ K. Matchev, ${ }^{157}$ N. Menendez, ${ }^{157}$ G. Mitselmakher, ${ }^{157}$ D. Rosenzweig, ${ }^{157}$ K. Shi, ${ }^{157}$ J. Wang, ${ }^{157}$ S. Wang, ${ }^{157}$ X. Zuo, ${ }^{157}$ Y. R. Joshi, ${ }^{158}$ S. Linn, ${ }^{158}$ T. Adams, ${ }^{159}$ A. Askew, ${ }^{159}$ S. Hagopian, ${ }^{159}$ V. Hagopian, ${ }^{159}$ K. F. Johnson, ${ }^{159}$ R. Khurana, ${ }^{159}$ T. Kolberg, ${ }^{159}$ G. Martinez, ${ }^{159}$ T. Perry, ${ }^{159}$ H. Prosper, ${ }^{159}$ C. Schiber ${ }^{159}$ R. Yohay, ${ }^{159}$ J. Zhang, ${ }^{159}$ M. M. Baarmand, ${ }^{160}$ V. Bhopatkar, ${ }^{160}$ M. Hohlmann, ${ }^{160}$ D. Noonan, ${ }^{160}$ M. Rahmani, ${ }^{160}$ M. Saunders ${ }^{160}$ F. Yumiceva, ${ }^{160}$ M. R. Adams, ${ }^{161}$ L. Apanasevich, ${ }^{161}$ D. Berry, ${ }^{161}$ R. R. Betts, ${ }^{161}$ R. Cavanaugh, ${ }^{161}$ X. Chen, ${ }^{161}$ S. Dittmer, ${ }^{161}$ O. Evdokimov, ${ }^{161}$ C. E. Gerber, ${ }^{161}$ D. A. Hangal, ${ }^{161}$ 
D. J. Hofman, ${ }^{161}$ K. Jung, ${ }^{161}$ C. Mills, ${ }^{161}$ T. Roy, ${ }^{161}$ M. B. Tonjes, ${ }^{161}$ N. Varelas, ${ }^{161}$ H. Wang, ${ }^{161}$ X. Wang, ${ }^{161}$ Z. Wu, ${ }^{161}$ M. Alhusseini, ${ }^{162}$ B. Bilki, ${ }^{162, \text { aaa }}$ W. Clarida, ${ }^{162}$ K. Dilsiz, ${ }^{162, r r r}$ S. Durgut, ${ }^{162}$ R. P. Gandrajula, ${ }^{162}$ M. Haytmyradov, ${ }^{162}$ V. Khristenko, ${ }^{162}$ O. K. Köseyan, ${ }^{162}$ J.-P. Merlo, ${ }^{162}$ A. Mestvirishvili, ${ }^{162}$ A. Moeller, ${ }^{162}$ J. Nachtman, ${ }^{162}$ H. Ogul, ${ }^{162, s s s}$ Y. Onel, ${ }^{162}$ F. Ozok, ${ }^{162, t t t}$ A. Penzo, ${ }^{162}$ C. Snyder, ${ }^{162}$ E. Tiras, ${ }^{162}$ J. Wetzel, ${ }^{162}$ B. Blumenfeld, ${ }^{163}$ A. Cocoros, ${ }^{163}$ N. Eminizer, ${ }^{163}$ D. Fehling, ${ }^{163}$ L. Feng, ${ }^{163}$ A. V. Gritsan, ${ }^{163}$ W. T. Hung, ${ }^{163}$ P. Maksimovic, ${ }^{163}$ J. Roskes, ${ }^{163}$ M. Swartz, ${ }^{163}$ M. Xiao, ${ }^{163}$ C. Baldenegro Barrera, ${ }^{164}$ P. Baringer, ${ }^{164}$ A. Bean, ${ }^{164}$ S. Boren, ${ }^{164}$ J. Bowen, ${ }^{164}$ A. Bylinkin, ${ }^{164}$ T. Isidori, ${ }^{164}$ S. Khalil,${ }^{164}$ J. King, ${ }^{164}$ A. Kropivnitskaya, ${ }^{164}$ C. Lindsey, ${ }^{164}$ D. Majumder ${ }^{164}$ W. Mcbrayer ${ }^{164}$ N. Minafra,${ }^{164}$ M. Murray, ${ }^{164}$ C. Rogan, ${ }^{164}$ C. Royon, ${ }^{164}$ S. Sanders, ${ }^{164}$ E. Schmitz, ${ }^{164}$ J. D. Tapia Takaki, ${ }^{164}$ Q. Wang, ${ }^{164}$ J. Williams, ${ }^{164}$ S. Duric, ${ }^{165}$ A. Ivanov, ${ }^{165}$ K. Kaadze, ${ }^{165}$ D. Kim, ${ }^{165}$ Y. Maravin, ${ }^{165}$ D. R. Mendis, ${ }^{165}$ T. Mitchell, ${ }^{165}$ A. Modak, ${ }^{165}$ A. Mohammadi, ${ }^{165}$ F. Rebassoo, ${ }^{166}$ D. Wright, ${ }^{166}$ A. Baden, ${ }^{167}$ O. Baron, ${ }^{167}$ A. Belloni, ${ }^{167}$ S. C. Eno, ${ }^{167}$ Y. Feng, ${ }^{167}$ C. Ferraioli, ${ }^{167}$ N. J. Hadley ${ }^{167}$ S. Jabeen, ${ }^{167}$ G. Y. Jeng, ${ }^{167}$ R. G. Kellogg, ${ }^{167}$ J. Kunkle, ${ }^{167}$ A. C. Mignerey, ${ }^{167}$ S. Nabili, ${ }^{167}$ F. Ricci-Tam, ${ }^{167}$ M. Seidel,${ }^{167}$ Y. H. Shin, ${ }^{167}$ A. Skuja ${ }^{167}$ S. C. Tonwar, ${ }^{167}$ K. Wong,${ }^{167}$ D. Abercrombie, ${ }^{168}$ B. Allen,${ }^{168}$ A. Baty, ${ }^{168}$ R. Bi, ${ }^{168}$ S. Brandt, ${ }^{168}$ W. Busza, ${ }^{168}$ I. A. Cali, ${ }^{168}$ M. D' Alfonso, ${ }^{168}$ G. Gomez Ceballos, ${ }^{168}$ M. Goncharov, ${ }^{168}$ P. Harris, ${ }^{168}$ D. Hsu, ${ }^{168}$ M. Hu, ${ }^{168}$ M. Klute, ${ }^{168}$ D. Kovalskyi, ${ }^{168}$ Y.-J. Lee, ${ }^{168}$ P. D. Luckey, ${ }^{168}$ B. Maier, ${ }^{168}$ A. C. Marini, ${ }^{168}$ C. Mcginn, ${ }^{168}$ C. Mironov, ${ }^{168}$ S. Narayanan, ${ }^{168}$ X. Niu, ${ }^{168}$ C. Paus, ${ }^{168}$ D. Rankin, ${ }^{168}$ C. Roland, ${ }^{168}$ G. Roland, ${ }^{168}$ Z. Shi, ${ }^{168}$ G. S. F. Stephans, ${ }^{168}$ K. Sumorok, ${ }^{168}$ K. Tatar,${ }^{168}$ D. Velicanu, ${ }^{168}$ J. Wang, ${ }^{168}$ T. W. Wang, ${ }^{168}$ B. Wyslouch,${ }^{168}$ A. C. Benvenuti, ${ }^{169, a}$ R. M. Chatterjee, ${ }^{169}$ A. Evans,${ }^{169}$ S. Guts, ${ }^{169}$ P. Hansen,${ }^{169}$ J. Hiltbrand, ${ }^{169}$ S. Kalafut ${ }^{169}$ Y. Kubota, ${ }^{169}$ Z. Lesko, ${ }^{169}$ J. Mans, ${ }^{169}$ R. Rusack, ${ }^{169}$ M. A. Wadud,${ }^{169}$ J. G. Acosta, ${ }^{170}$ S. Oliveros, ${ }^{170}$ E. Avdeeva, ${ }^{171}$ K. Bloom, ${ }^{171}$ D. R. Claes, ${ }^{171}$ C. Fangmeier, ${ }^{171}$ L. Finco, ${ }^{171}$ F. Golf, ${ }^{171}$ R. Gonzalez Suarez, ${ }^{171}$ R. Kamalieddin, ${ }^{171}$ I. Kravchenko, ${ }^{171}$ J. E. Siado, ${ }^{171}$ G. R. Snow, ${ }^{171}$ B. Stieger, ${ }^{171}$ A. Godshalk, ${ }^{172}$ C. Harrington, ${ }^{172}$ I. Iashvili, ${ }^{172}$ A. Kharchilava, ${ }^{172}$ C. Mclean, ${ }^{172}$ D. Nguyen, ${ }^{172}$ A. Parker, ${ }^{172}$ S. Rappoccio, ${ }^{172}$ B. Roozbahani, ${ }^{172}$ G. Alverson, ${ }^{173}$ E. Barberis, ${ }^{173}$ C. Freer, ${ }^{173}$ Y. Haddad, ${ }^{173}$ A. Hortiangtham, ${ }^{173}$ G. Madigan, ${ }^{173}$ D. M. Morse, ${ }^{173}$ T. Orimoto, ${ }^{173}$ L. Skinnari, ${ }^{173}$ A. Tishelman-Charny, ${ }^{173}$

T. Wamorkar, ${ }^{173}$ B. Wang, ${ }^{173}$ A. Wisecarver, ${ }^{173}$ D. Wood, ${ }^{173}$ S. Bhattacharya, ${ }^{174}$ J. Bueghly, ${ }^{174}$ T. Gunter, ${ }^{174}$ K. A. Hahn, ${ }^{174}$ N. Odell, ${ }^{174}$ M. H. Schmitt, ${ }^{174}$ K. Sung, ${ }^{174}$ M. Trovato, ${ }^{174}$ M. Velasco, ${ }^{174}$ R. Bucci, ${ }^{175}$ N. Dev, ${ }^{175}$ R. Goldouzian, ${ }^{175}$ M. Hildreth, ${ }^{175}$ K. Hurtado Anampa, ${ }^{175}$ C. Jessop, ${ }^{175}$ D. J. Karmgard, ${ }^{175}$ K. Lannon, ${ }^{175}$ W. Li, ${ }^{175}$ N. Loukas, ${ }^{175}$ N. Marinelli, ${ }^{175}$ I. Mcalister, ${ }^{175}$ F. Meng, ${ }^{175}$ C. Mueller, ${ }^{175}$ Y. Musienko,${ }^{175, k k}$ M. Planer, ${ }^{175}$ R. Ruchti, ${ }^{175}$ P. Siddireddy, ${ }^{175}$ G. Smith, ${ }^{175}$ S. Taroni, ${ }^{175}$ M. Wayne, ${ }^{175}$ A. Wightman, ${ }^{175}$ M. Wolf, ${ }^{175}$ A. Woodard, ${ }^{175}$ J. Alimena, ${ }^{176}$ B. Bylsma, ${ }^{176}$

L. S. Durkin, ${ }^{176}$ S. Flowers, ${ }^{176}$ B. Francis, ${ }^{176}$ C. Hill, ${ }^{176}$ W. Ji, ${ }^{176}$ A. Lefeld, ${ }^{176}$ T. Y. Ling, ${ }^{176}$ B. L. Winer, ${ }^{176}$ S. Cooperstein, ${ }^{177}$ G. Dezoort, ${ }^{177}$ P. Elmer, ${ }^{177}$ J. Hardenbrook, ${ }^{177}$ N. Haubrich, ${ }^{177}$ S. Higginbotham, ${ }^{177}$

A. Kalogeropoulos, ${ }^{177}$ S. Kwan, ${ }^{177}$ D. Lange, ${ }^{177}$ M. T. Lucchini, ${ }^{177}$ J. Luo,${ }^{177}$ D. Marlow, ${ }^{177}$ K. Mei, ${ }^{177}$ I. Ojalvo, ${ }^{177}$ J. Olsen, ${ }^{177}$ C. Palmer, ${ }^{177}$ P. Piroué, ${ }^{177}$ J. Salfeld-Nebgen, ${ }^{177}$ D. Stickland, ${ }^{177}$ C. Tully, ${ }^{177}$ Z. Wang, ${ }^{177}$ S. Malik, ${ }^{178}$ S. Norberg, ${ }^{178}$ A. Barker, ${ }^{179}$ V. E. Barnes, ${ }^{179}$ S. Das, ${ }^{179}$ L. Gutay, ${ }^{179}$ M. Jones, ${ }^{179}$ A. W. Jung, ${ }^{179}$ A. Khatiwada, ${ }^{179}$ B. Mahakud, ${ }^{179}$ D. H. Miller, ${ }^{179}$ G. Negro, ${ }^{179}$ N. Neumeister, ${ }^{179}$ C. C. Peng, ${ }^{179}$ S. Piperov,${ }^{179}$ H. Qiu, ${ }^{179}$ J. F. Schulte, ${ }^{179}$ J. Sun, ${ }^{179}$ F. Wang, ${ }^{179}$ R. Xiao, ${ }^{179}$ W. Xie, ${ }^{179}$ T. Cheng, ${ }^{180}$ J. Dolen, ${ }^{180}$ N. Parashar, ${ }^{180}$ K. M. Ecklund, ${ }^{181}$ S. Freed, ${ }^{181}$ F. J. M. Geurts, ${ }^{181}$ M. Kilpatrick, ${ }^{181}$ Arun Kumar,${ }^{181}$ W. Li, ${ }^{181}$ B. P. Padley,${ }^{181}$ R. Redjimi, ${ }^{181}$ J. Roberts, ${ }^{181}$ J. Rorie, ${ }^{181}$ W. Shi, ${ }^{181}$ A. G. Stahl Leiton, ${ }^{181}$ Z. Tu, ${ }^{181}$ A. Zhang, ${ }^{181}$ A. Bodek, ${ }^{182}$ P. de Barbaro, ${ }^{182}$ R. Demina, ${ }^{182}$ Y. t. Duh, ${ }^{182}$

J. L. Dulemba, ${ }^{182}$ C. Fallon, ${ }^{182}$ T. Ferbel, ${ }^{182}$ M. Galanti, ${ }^{182}$ A. Garcia-Bellido, ${ }^{182}$ J. Han,${ }^{182}$ O. Hindrichs, ${ }^{182}$

A. Khukhunaishvili, ${ }^{182}$ E. Ranken, ${ }^{182}$ P. Tan, ${ }^{182}$ R. Taus, ${ }^{182}$ B. Chiarito, ${ }^{183}$ J. P. Chou, ${ }^{183}$ A. Gandrakota, ${ }^{183}$ Y. Gershtein, ${ }^{183}$ E. Halkiadakis, ${ }^{183}$ A. Hart, ${ }^{183}$ M. Heindl,${ }^{183}$ E. Hughes, ${ }^{183}$ S. Kaplan, ${ }^{183}$ S. Kyriacou, ${ }^{183}$ I. Laflotte ${ }^{183}$ A. Lath ${ }^{183}$ R. Montalvo, ${ }^{183}$ K. Nash, ${ }^{183}$ M. Osherson, ${ }^{183}$ H. Saka, ${ }^{183}$ S. Salur, ${ }^{183}$ S. Schnetzer, ${ }^{183}$ D. Sheffield, ${ }^{183}$ S. Somalwar, ${ }^{183}$ R. Stone ${ }^{183}$ S. Thomas, ${ }^{183}$ P. Thomassen, ${ }^{183}$ H. Acharya, ${ }^{184}$ A. G. Delannoy, ${ }^{184}$ J. Heideman, ${ }^{184}$ G. Riley, ${ }^{184}$ S. Spanier, ${ }^{184}$ O. Bouhali, ${ }^{185, \text { uuu }}$ A. Celik, ${ }^{185}$ M. Dalchenko, ${ }^{185}$ M. De Mattia, ${ }^{185}$ A. Delgado, ${ }^{185}$ S. Dildick, ${ }^{185}$ R. Eusebi, ${ }^{185}$ J. Gilmore, ${ }^{185}$

T. Huang, ${ }^{185}$ T. Kamon, ${ }^{185, \mathrm{vvv}}$ S. Luo, ${ }^{185}$ D. Marley, ${ }^{185}$ R. Mueller, ${ }^{185}$ D. Overton, ${ }^{185}$ L. Perniè, ${ }^{185}$ D. Rathjens, ${ }^{185}$ A. Safonov, ${ }^{185}$ N. Akchurin, ${ }^{186}$ J. Damgov, ${ }^{186}$ F. De Guio, ${ }^{186}$ S. Kunori, ${ }^{186}$ K. Lamichhane, ${ }^{186}$ S. W. Lee,${ }^{186}$ T. Mengke, ${ }^{186}$ S. Muthumuni, ${ }^{186}$ T. Peltola, ${ }^{186}$ S. Undleeb, ${ }^{186}$ I. Volobouev, ${ }^{186}$ Z. Wang, ${ }^{186}$ A. Whitbeck, ${ }^{186}$ S. Greene, ${ }^{187}$ A. Gurrola, ${ }^{187}$ R. Janjam, ${ }^{187}$ W. Johns, ${ }^{187}$ C. Maguire, ${ }^{187}$ A. Melo, ${ }^{187} \mathrm{H}$. Ni ${ }^{187}$ K. Padeken, ${ }^{187}$ F. Romeo,${ }^{187}$ P. Sheldon, ${ }^{187}$ S. Tuo, ${ }^{187}$ J. Velkovska, ${ }^{187}$ M. Verweij, ${ }^{187}$ M. W. Arenton, ${ }^{188}$ P. Barria, ${ }^{188}$ B. Cox ${ }^{188}$ G. Cummings, ${ }^{188}$ R. Hirosky, ${ }^{188}$ M. Joyce, ${ }^{188}$ A. Ledovskoy, ${ }^{188}$ C. Neu ${ }^{188}$ B. Tannenwald ${ }^{188}$ Y. Wang, ${ }^{188}$ E. Wolfe, ${ }^{188}$ F. Xia, ${ }^{188}$ R. Harr, ${ }^{189}$ P. E. Karchin, ${ }^{189}$ N. Poudyal, ${ }^{189}$ J. Sturdy, ${ }^{189}$ P. Thapa ${ }^{189}$ S. Zaleski, ${ }^{189}$ J. Buchanan, ${ }^{190}$ C. Caillol, ${ }^{190}$ D. Carlsmith, ${ }^{190}$ S. Dasu, ${ }^{190}$ 
I. De Bruyn, ${ }^{190}$ L. Dodd, ${ }^{190}$ B. Gomber, ${ }^{190, w w w}$ M. Grothe, ${ }^{190}$ M. Herndon, ${ }^{190}$ A. Hervé, ${ }^{190}$ U. Hussain, ${ }^{190}$ P. Klabbers, ${ }^{190}$ A. Lanaro, ${ }^{190}$ K. Long, ${ }^{190}$ R. Loveless,${ }^{190}$ T. Ruggles, ${ }^{190}$ A. Savin,,${ }^{190}$ V. Sharma, ${ }^{190}$ W. H. Smith, ${ }^{190}$ and N. Woods ${ }^{190}$

(CMS Collaboration)

\footnotetext{
${ }^{1}$ Yerevan Physics Institute, Yerevan, Armenia

${ }^{2}$ Institut für Hochenergiephysik, Wien, Austria

${ }^{3}$ Institute for Nuclear Problems, Minsk, Belarus

${ }^{4}$ Universiteit Antwerpen, Antwerpen, Belgium

${ }^{5}$ Vrije Universiteit Brussel, Brussel, Belgium

${ }^{6}$ Université Libre de Bruxelles, Bruxelles, Belgium

${ }^{7}$ Ghent University, Ghent, Belgium

${ }^{8}$ Université Catholique de Louvain, Louvain-la-Neuve, Belgium

${ }^{9}$ Centro Brasileiro de Pesquisas Fisicas, Rio de Janeiro, Brazil

${ }^{10}$ Universidade do Estado do Rio de Janeiro, Rio de Janeiro, Brazil

${ }^{11 a}$ Universidade Estadual Paulista, São Paulo, Brazil

${ }^{11 \mathrm{~b}}$ Universidade Federal do ABC, São Paulo, Brazil

${ }^{12}$ Institute for Nuclear Research and Nuclear Energy, Bulgarian Academy of Sciences, Sofia, Bulgaria

${ }^{13}$ University of Sofia, Sofia, Bulgaria

${ }^{14}$ Beihang University, Beijing, China

${ }^{15}$ Institute of High Energy Physics, Beijing, China

${ }^{16}$ State Key Laboratory of Nuclear Physics and Technology, Peking University, Beijing, China

${ }^{17}$ Tsinghua University, Beijing, China

${ }^{18}$ Universidad de Los Andes, Bogota, Colombia

${ }^{19}$ Universidad de Antioquia, Medellin, Colombia

${ }^{20}$ University of Split, Faculty of Electrical Engineering, Mechanical Engineering and Naval Architecture, Split, Croatia

${ }^{21}$ University of Split, Faculty of Science, Split, Croatia

${ }^{22}$ Institute Rudjer Boskovic, Zagreb, Croatia

${ }^{23}$ University of Cyprus, Nicosia, Cyprus

${ }^{24}$ Charles University, Prague, Czech Republic

${ }^{25}$ Escuela Politecnica Nacional, Quito, Ecuador

${ }^{26}$ Universidad San Francisco de Quito, Quito, Ecuador

${ }^{27}$ Academy of Scientific Research and Technology of the Arab Republic of Egypt,

Egyptian Network of High Energy Physics, Cairo, Egypt

${ }^{28}$ National Institute of Chemical Physics and Biophysics, Tallinn, Estonia

${ }^{29}$ Department of Physics, University of Helsinki, Helsinki, Finland

${ }^{30}$ Helsinki Institute of Physics, Helsinki, Finland

${ }^{31}$ Lappeenranta University of Technology, Lappeenranta, Finland

${ }^{32} I R F U, C E A$, Université Paris-Saclay, Gif-sur-Yvette, France

${ }^{33}$ Laboratoire Leprince-Ringuet, Ecole polytechnique, CNRS/IN2P3, Université Paris-Saclay, Palaiseau, France

${ }^{34}$ Université de Strasbourg, CNRS, IPHC UMR 7178, Strasbourg, France

${ }^{35}$ Centre de Calcul de l'Institut National de Physique Nucleaire et de Physique des Particules, CNRS/IN2P3, Villeurbanne, France

${ }^{36}$ Université de Lyon, Université Claude Bernard Lyon 1, CNRS-IN2P3, Institut de Physique Nucléaire de Lyon, Villeurbanne, France

${ }^{37}$ Georgian Technical University, Tbilisi, Georgia

${ }^{38}$ Tbilisi State University, Tbilisi, Georgia

${ }^{39}$ RWTH Aachen University, I. Physikalisches Institut, Aachen, Germany

${ }^{40}$ RWTH Aachen University, III. Physikalisches Institut A, Aachen, Germany

${ }^{41}$ RWTH Aachen University, III. Physikalisches Institut B, Aachen, Germany

${ }^{42}$ Deutsches Elektronen-Synchrotron, Hamburg, Germany

${ }^{43}$ University of Hamburg, Hamburg, Germany

${ }^{44}$ Karlsruher Institut fuer Technologie, Karlsruhe, Germany

${ }^{45}$ Institute of Nuclear and Particle Physics (INPP), NCSR Demokritos, Aghia Paraskevi, Greece

${ }^{46}$ National and Kapodistrian University of Athens, Athens, Greece

${ }^{47}$ National Technical University of Athens, Athens, Greece

${ }^{48}$ University of Ioánnina, Ioánnina, Greece

${ }^{49}$ MTA-ELTE Lendület CMS Particle and Nuclear Physics Group, Eötvös Loránd University, Budapest, Hungary

${ }^{50}$ Wigner Research Centre for Physics, Budapest, Hungary

${ }^{51}$ Institute of Nuclear Research ATOMKI, Debrecen, Hungary
} 


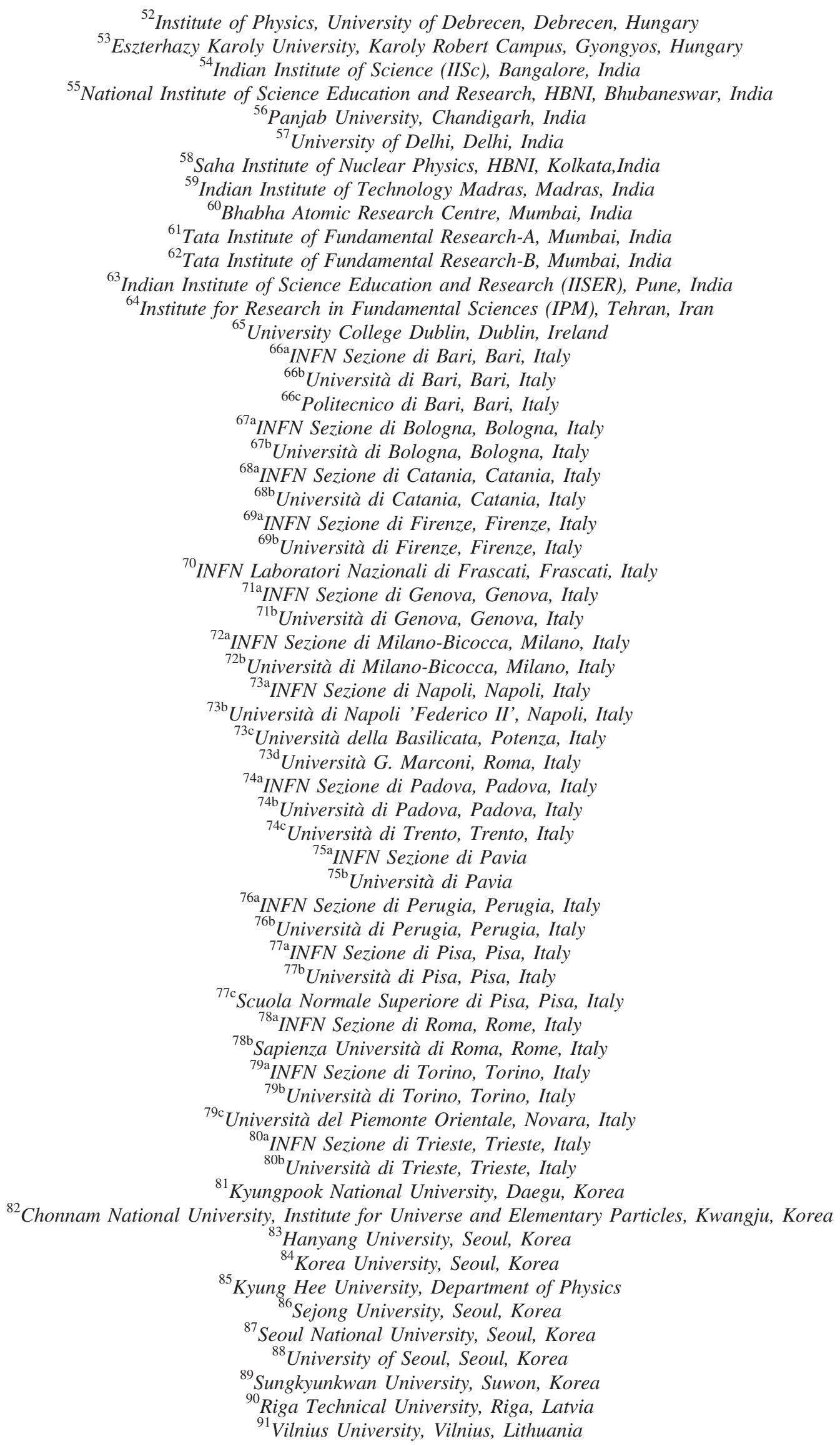


${ }^{92}$ National Centre for Particle Physics, Universiti Malaya, Kuala Lumpur, Malaysia

${ }^{93}$ Universidad de Sonora (UNISON), Hermosillo, Mexico

${ }^{94}$ Centro de Investigacion y de Estudios Avanzados del IPN, Mexico City, Mexico

${ }^{95}$ Universidad Iberoamericana, Mexico City, Mexico

${ }^{96}$ Benemerita Universidad Autonoma de Puebla, Puebla, Mexico

${ }^{97}$ Universidad Autónoma de San Luis Potosí, San Luis Potosí, Mexico

${ }^{98}$ University of Montenegro, Podgorica, Montenegro

${ }^{99}$ University of Auckland, Auckland, New Zealand

${ }^{100}$ University of Canterbury, Christchurch, New Zealand

${ }^{101}$ National Centre for Physics, Quaid-I-Azam University, Islamabad, Pakistan

${ }^{102}$ AGH University of Science and Technology Faculty of Computer Science, Electronics and Telecommunications, Krakow, Poland

${ }^{103}$ National Centre for Nuclear Research, Swierk, Poland

${ }^{104}$ Institute of Experimental Physics, Faculty of Physics, University of Warsaw, Warsaw, Poland

${ }^{105}$ Laboratório de Instrumentação e Física Experimental de Partículas, Lisboa, Portugal

${ }^{106}$ Joint Institute for Nuclear Research, Dubna, Russia

${ }^{107}$ Petersburg Nuclear Physics Institute, Gatchina (St. Petersburg), Russia

${ }^{108}$ Institute for Nuclear Research, Moscow, Russia

${ }^{109}$ Institute for Theoretical and Experimental Physics named by A.I.Alikhanov of NRC «Kurchatov Institute», Moscow, Russia

${ }^{110}$ Moscow Institute of Physics and Technology, Moscow, Russia

${ }^{111}$ National Research Nuclear University 'Moscow Engineering Physics Institute' (MEPhI), Moscow, Russia

${ }^{112}$ P.N. Lebedev Physical Institute, Moscow, Russia

${ }^{113}$ Skobeltsyn Institute of Nuclear Physics, Lomonosov Moscow State University, Moscow, Russia

${ }^{114}$ Novosibirsk State University (NSU), Novosibirsk, Russia

${ }^{115}$ Institute for High Energy Physics of National Research Centre 'Kurchatov Institute', Protvino, Russia

${ }^{116}$ National Research Tomsk Polytechnic University, Tomsk, Russia

${ }^{117}$ Tomsk State University, Tomsk, Russia

${ }^{118}$ University of Belgrade: Faculty of Physics and VINCA Institute of Nuclear Sciences, Belgrade, Serbia

${ }^{119}$ Centro de Investigaciones Energéticas Medioambientales y Tecnológicas (CIEMAT), Madrid, Spain

${ }^{120}$ Universidad Autónoma de Madrid, Madrid, Spain

${ }^{121}$ Universidad de Oviedo, Oviedo, Spain

${ }^{122}$ Instituto de Física de Cantabria (IFCA), CSIC-Universidad de Cantabria, Santander, Spain

${ }^{123}$ University of Colombo, Colombo, Sri Lanka

${ }^{124}$ University of Ruhuna, Department of Physics, Matara, Sri Lanka

${ }^{125}$ CERN, European Organization for Nuclear Research, Geneva, Switzerland

${ }^{126}$ Paul Scherrer Institut, Villigen, Switzerland

${ }^{127}$ ETH Zurich-Institute for Particle Physics and Astrophysics (IPA), Zurich, Switzerland

${ }^{128}$ Universität Zürich, Zurich, Switzerland

${ }^{129}$ National Central University, Chung-Li, Taiwan

${ }^{130}$ National Taiwan University (NTU), Taipei, Taiwan

${ }^{131}$ Chulalongkorn University, Faculty of Science, Department of Physics, Bangkok, Thailand

${ }^{132}$ Cukurova University, Physics Department, Science and Art Faculty, Adana, Turkey

${ }^{133}$ Middle East Technical University, Physics Department, Ankara, Turkey

${ }^{134}$ Bogazici University, Istanbul, Turkey

${ }^{135}$ Istanbul Technical University, Istanbul, Turkey

${ }^{136}$ Institute for Scintillation Materials of National Academy of Science of Ukraine, Kharkov, Ukraine

${ }^{137}$ National Scientific Center, Kharkov Institute of Physics and Technology, Kharkov, Ukraine

${ }^{138}$ University of Bristol, Bristol, United Kingdom

${ }^{139}$ Rutherford Appleton Laboratory, Didcot, United Kingdom

${ }^{140}$ Imperial College, London, United Kingdom

${ }^{141}$ Brunel University, Uxbridge, United Kingdom

${ }^{142}$ Baylor University, Waco, Texas, USA

${ }^{143}$ Catholic University of America, Washington, DC, USA

${ }^{144}$ The University of Alabama, Tuscaloosa, Alabama, USA

${ }^{145}$ Boston University, Boston, Massachusetts, USA

${ }^{146}$ Brown University, Providence, Rhode Island, USA

${ }^{147}$ University of California, Davis, Davis, California, USA

${ }^{148}$ University of California, Los Angeles, California, USA

${ }^{149}$ University of California, Riverside, Riverside, California, USA

${ }^{150}$ University of California, San Diego, La Jolla, California, USA

${ }^{151}$ University of California, Santa Barbara-Department of Physics, Santa Barbara, California, USA 
${ }^{152}$ California Institute of Technology, Pasadena, California, USA

${ }^{153}$ Carnegie Mellon University, Pittsburgh, Pennsylvania, USA

${ }^{154}$ University of Colorado Boulder, Boulder, Colorado, USA

${ }^{155}$ Cornell University, Ithaca, New York, USA

${ }^{156}$ Fermi National Accelerator Laboratory, Batavia, Illinois, USA

${ }^{157}$ University of Florida, Gainesville, Florida, USA

${ }^{158}$ Florida International University, Miami, Florida, USA

${ }^{159}$ Florida State University, Tallahassee, Florida, USA

${ }^{160}$ Florida Institute of Technology, Melbourne, Florida, USA

${ }^{161}$ University of Illinois at Chicago (UIC), Chicago, Illinois, USA

${ }^{162}$ The University of Iowa, Iowa City, Iowa, USA

${ }^{163}$ Johns Hopkins University, Baltimore, Maryland, USA

${ }^{164}$ The University of Kansas, Lawrence, Kansas, USA

${ }^{165}$ Kansas State University, Manhattan, Kansas, USA

${ }^{166}$ Lawrence Livermore National Laboratory, Livermore, California, USA

${ }^{167}$ University of Maryland, College Park, Maryland, USA

${ }^{168}$ Massachusetts Institute of Technology, Cambridge, Massachusetts, USA

${ }^{169}$ University of Minnesota, Minneapolis, Minnesota, USA

${ }^{170}$ University of Mississippi, Oxford, Mississippi, USA

${ }^{171}$ University of Nebraska-Lincoln, Lincoln, Nebraska, USA

${ }^{172}$ State University of New York at Buffalo, Buffalo, New York, USA

${ }^{173}$ Northeastern University, Boston, Massachusetts, USA

${ }^{174}$ Northwestern University, Evanston, Illinois, USA

${ }^{175}$ University of Notre Dame, Notre Dame, Indiana, USA

${ }^{176}$ The Ohio State University, Columbus, Ohio, USA

${ }^{177}$ Princeton University, Princeton, New Jersey, USA

${ }^{178}$ University of Puerto Rico, Mayaguez, Puerto Rico, USA

${ }^{179}$ Purdue University, West Lafayette, Indiana, USA

${ }^{180}$ Purdue University Northwest, Hammond, Indiana, USA

${ }^{181}$ Rice University, Houston, Texas, USA

${ }^{182}$ University of Rochester, Rochester, New York, USA

${ }^{183}$ Rutgers, The State University of New Jersey, Piscataway, New Jersey, USA

${ }^{184}$ University of Tennessee, Knoxville, Tennessee, USA

${ }^{185}$ Texas A\&M University, College Station, Texas, USA

${ }^{186}$ Texas Tech University, Lubbock, Texas, USA

${ }^{187}$ Vanderbilt University, Nashville, Tennessee, USA

${ }^{188}$ University of Virginia, Charlottesville, Virginia, USA

${ }^{189}$ Wayne State University, Detroit, Michigan, USA

${ }^{190}$ University of Wisconsin-Madison, Madison, Wisconsin, USA

${ }^{\mathrm{a}}$ Deceased.

${ }^{\mathrm{b}}$ Also at Vienna University of Technology, Vienna, Austria.

${ }^{\mathrm{c}}$ Also at IRFU, CEA, Université Paris-Saclay, Gif-sur-Yvette, France.

${ }^{\mathrm{d}}$ Also at Universidade Estadual de Campinas, Campinas, Brazil.

${ }^{\mathrm{e}}$ Also at Federal University of Rio Grande do Sul, Porto Alegre, Brazil.

${ }^{\mathrm{f}}$ Also at Universidade Federal de Pelotas, Pelotas, Brazil.

${ }^{\mathrm{g}}$ Also at Université Libre de Bruxelles, Bruxelles, Belgium.

${ }^{\mathrm{h}}$ Also at University of Chinese Academy of Sciences, Bejing, China.

${ }^{\mathrm{i}}$ Also at Institute for Theoretical and Experimental Physics named by A.I.Alikhanov of NRC «Kurchatov Institute», Moscow, Russia.

${ }^{\mathrm{j}}$ Also at Joint Institute for Nuclear Research, Dubna, Russia.

${ }^{\mathrm{k}}$ Also at British University in Egypt, Cairo, Egypt.

${ }^{1}$ Also at Suez University, Suez, Egypt.

${ }^{\mathrm{m}}$ Also at Purdue University, West Lafayette, Indiana, USA.

${ }^{\mathrm{n}}$ Also at Université de Haute Alsace, Mulhouse, France.

${ }^{\circ}$ Also at Tbilisi State University, Tbilisi, Georgia.

${ }^{\mathrm{p}}$ Also at CERN, European Organization for Nuclear Research, Geneva, Switzerland.

${ }^{\mathrm{q}}$ Also at RWTH Aachen University, III. Physikalisches Institut A, Aachen, Germany.

${ }^{\mathrm{r}}$ Also at University of Hamburg, Hamburg, Germany.

${ }^{\mathrm{s}}$ Also at Brandenburg University of Technology, Cottbus, Germany. 


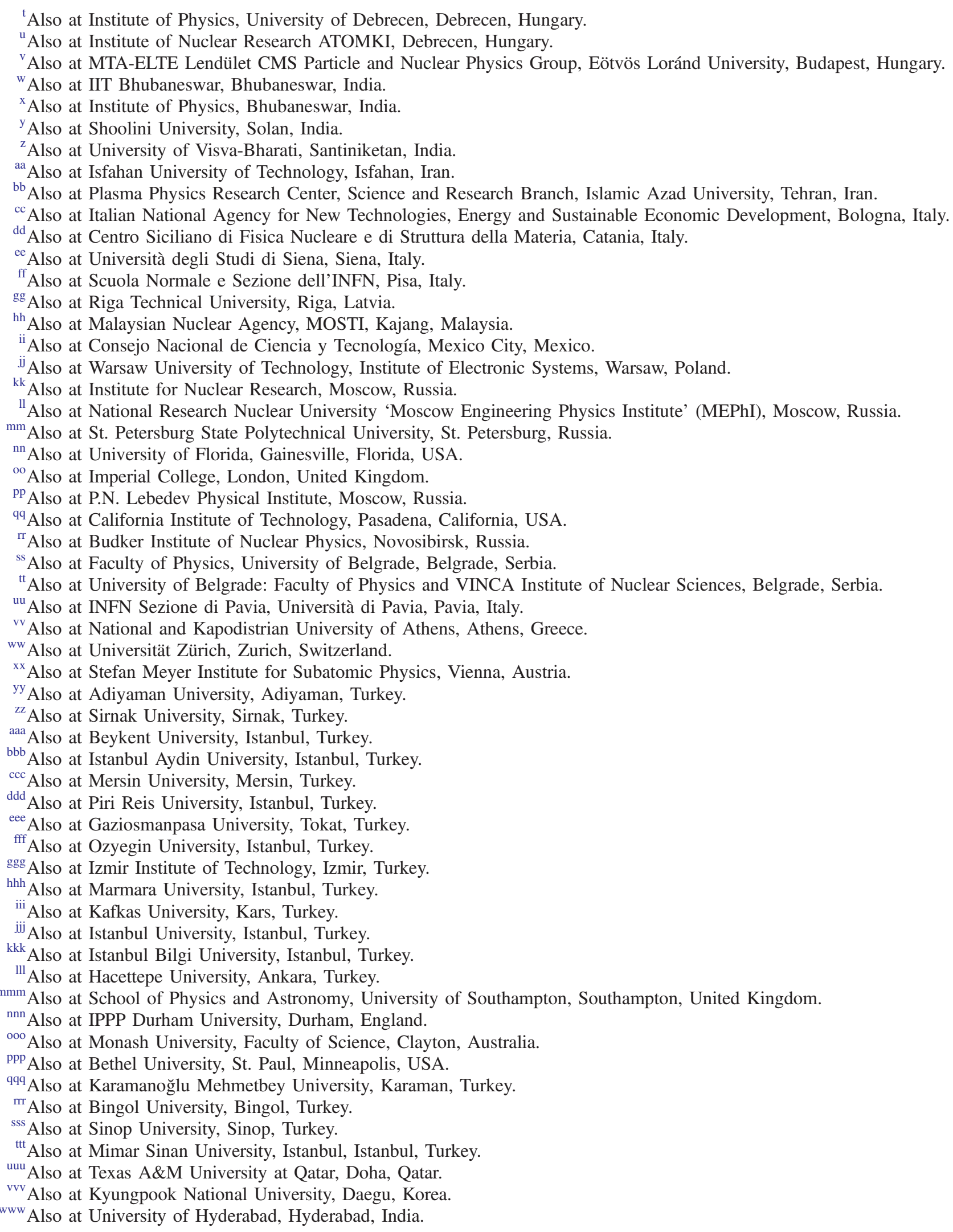

Also at Institute of Physics, University of Debrecen, Debrecen, Hungary.

Also at MTA-ELTE Lendület CMS Particle and Nuclear Physics Group, Eötvös Loránd University, Budapest, Hungary.

${ }^{\mathrm{w}}$ Also at IIT Bhubaneswar, Bhubaneswar, India.

"Also at Institute of Physics, Bhubaneswar, India.

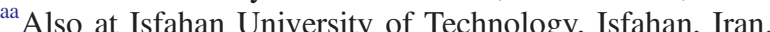

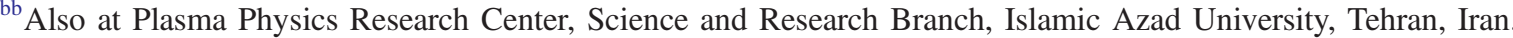

Also at Italian National Agency for New Technologies, Energy and Sustainable Economic Development, Bologna, Italy.

${ }^{e e}$ Also at Università degli Studi di Siena, Siena, Italy.

${ }^{\mathrm{ff}}$ Also at Scuola Normale e Sezione dell'INFN, Pisa, Italy.

Also at Riga Technical University, Riga, Latvia.

Also at Malaysian Nuclear Agency, MOSTI, Kajang, Malaysia.

${ }^{\mathrm{k}}$ Also at Institute for Nuclear Research, Moscow, Russia.

"Also at National Research Nuclear University 'Moscow Engineering Physics Institute' (MEPhI), Moscow, Russia.

${ }^{\mathrm{nm}}$ Also at St. Petersburg State Polytechnical University, St. Petersburg, Russia

${ }^{\mathrm{nn}}$ Also at University of Florida, Gainesville, Florida, USA

Also at Imperial College, London, United Kingdom.

Also at P.N. Lebedev Physical Institute, Moscow, Russia.

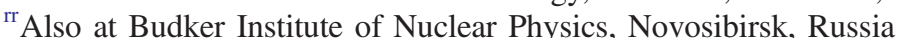

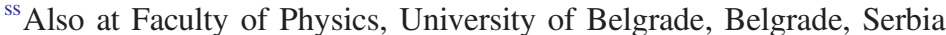

"Also at University of Belgrade: Faculty of Physics and VINCA Institute of Nuclear Sciences, Belgrade, Serbia.

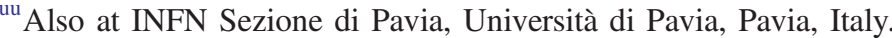

${ }^{v v}$ Also at National and Kapodistrian University of Athens, Athens, Greece.

${ }^{\mathrm{ww}}$ Also at Universität Zürich, Zurich, Switzerland.

${ }^{\mathrm{x}}$ Also at Stefan Meyer Institute for Subatomic Physics, Vienna, Austria.

. Turkey.

${ }^{\mathrm{ccc}}$ Also at Mersin University, Mersin, Turkey.

ddd Also at Piri Reis University, Istanbul, Turkey.

${ }^{e e e}$ Also at Gaziosmanpasa University, Tokat, Turkey.

Also at Ozyegin University, Istanbul, Turkey.

hhh Also at Marmara University, Istanbul, Turkey.

iit Also at Kafkas University, Kars, Turkey.

Also at Istanbul University, Istanbul, Turkey.

${ }^{k \mathrm{kk}}$ Also at Istanbul Bilgi University, Istanbul, Turkey.

${ }^{111}$ Also at Hacettepe University, Ankara, Turkey.

${ }^{\mathrm{nmm}}$ Also at School of Physics and Astronomy, University of Southampton, Southampton, United Kingdom.

${ }^{\mathrm{nnn}}$ Also at IPPP Durham University, Durham, England.

Also at Monash University, Faculty of Science, Clayton, Australia.

Aniversity, St. Paul, Minneapolis, USA.

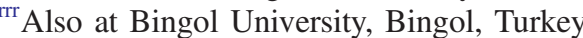

${ }^{\text {sss }}$ Also at Sinop University, Sinop, Turkey.

${ }^{\text {ttt }}$ Also at Mimar Sinan University, Istanbul, Istanbul, Turkey.

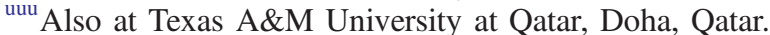

${ }^{w w w}$ Also at University of Hyderabad, Hyderabad, India. 